\title{
Surface waves in a square container due to its resonant horizontal elliptic motion
}

\author{
$\operatorname{AUTHOR}(\mathrm{S})$ :
}

Hiramitsu, Ai; Funakoshi, Mitsuaki

\section{CITATION:}

Hiramitsu, Ai ... [et al]. Surface waves in a square container due to its resonant horizontal elliptic motion. Fluid Dynamics Research 2015, 47(4): 45504.

\section{ISSUE DATE:}

2015-06-18

URL:

http://hdl.handle.net/2433/201634

\section{RIGHT:}

(c) 2015 The Japan Society of Fluid Mechanics and IOP Publishing Ltd.; This is an author-created, un-copyedited version of an article accepted for publication in 'Fluid Dynamics Research'. The publisher is not responsible for any errors or omissions in this version of the manuscript or any version derived from it. The Version of Record is available online at http://dx.doi.org/10.1088/0169-5983/47/4/045504:; この論文は出版社版でありません。引用の際には出版社版をご確 認ざ利用ください。; This is not the published version. Please cite only the published version. 


\title{
Surface waves in a square container due to its resonant horizontal elliptic motion
}

\author{
Ai Hiramitsu ${ }^{1}$ and Mitsuaki Funakoshi ${ }^{2} \ddagger$ \\ Department of Applied Analysis and Complex Dynamical Systems, Graduate School \\ of Informatics, Kyoto University, Yoshida-Honmachi, Kyoto, 606-8501, Japan \\ E-mail: ${ }^{1}$ ai_hiramitsu@acs.i.kyoto-u.ac.jp, ${ }^{2}$ mitsu@acs.i.kyoto-u.ac.jp
}

\begin{abstract}
Surface waves in a square container due to its resonant horizontal elliptic or linear motion are investigated theoretically. The motion of the container is characterized by the ratio, expressed as $\tan \phi$, of the length of the minor axis to the length of the major axis of its elliptic orbit, and by the angle $\theta$ between the directions of the major axis and one of its sidewalls. Using the reductive perturbation method, nonlinear time evolution equations for the complex amplitudes of two degenerate modes excited by this motion are derived with the inclusion of linear damping. When $\tan \phi$ is small, for any $\theta$ these equations have two kinds of stable stationary solutions corresponding to regular co-rotating waves whose direction of rotation is the same as that of the container, and regular counter-rotating waves of the opposite direction of rotation. As $\tan \phi$ increases to one, the region of forcing frequency in which stable regular counter-rotating waves are observed shrinks and then disappears for any $\theta$. Solutions with chaotic or periodic slow variations in amplitude and phase of excited surface waves are also obtained for forcing frequencies where no stable stationary solutions exist. Non-stationary solutions are either uni-directionally or bi-directionally rotating waves. For $\theta=0^{\circ}$, chaotic waves and bi-directionally rotating waves are observed more frequently for smaller $\tan \phi$. For $\theta=\phi=0^{\circ}$, for sufficiently small fluid depth, regular non-rotating waves are expected to occur for any forcing frequency. Moreover, stable stationary and non-stationary solutions obtained for $\phi=0^{\circ}$ are found to agree fairly well with the experimental results in a preceding study.
\end{abstract}

Keywords: surface wave, sloshing, elliptic motion, rotating wave 


\section{Introduction}

When a container partially filled with a liquid is oscillated resonantly, surface waves of large amplitude are excited even if the amplitude of oscillation is small. Therefore, because of their interesting behavior as an example of nonlinear dynamical systems and their importance in many applications, surface waves due to the resonant oscillation of a container have been investigated theoretically and experimentally by many researchers (see for example Miles and Henderson 1990, Ibrahim 2005, Faltinsen and Timokha 2009). In these studies, a container is oscillated in various ways such as vertical, horizontal, and pitching oscillations.

There are several studies on surface waves due to a resonant horizontal linear oscillation in which a cylindrical container is oscillated with a frequency close to the natural frequency of a few surface-wave modes. In his experiments on surface waves due to this type of oscillation of a circular container, Hutton (1963) found two kinds of regular wave motions, rotating and non-rotating (planar) waves. Miles (1984) derived nonlinear equations that govern the time evolution of complex amplitudes of two degenerate modes in a circular container excited by its resonant horizontal linear oscillations. He also showed that his equations have chaotic and periodic solutions as well as two kinds of stationary solutions corresponding to the above regular wave motions. Funakoshi and Inoue (1988) performed experiments on the same problem, and showed that the solutions of Miles' equations agree well with their experimental results on regular rotating and non-rotating waves and on waves of chaotic or periodic amplitude modulations. Funakoshi and Inoue (1990) also observed the behavior of excited surface waves corresponding to period-doubling, symmetry-breaking and homoclinic bifurcations in their experiments on the same problem. Nobili et al (1988) observed the route to chaos through period-doubling bifurcations in a similar experiment. RoyonLebeaud et al (2007) also examined mainly surface waves generated by the horizontal oscillation of circular and square-base containers experimentally. In experiments over a relatively wide range of forcing amplitude, they observed breaking of excited waves as well as regular rotating and non-rotating waves and waves of chaotic amplitude modulations.

Yoshimatsu and Funakoshi (2001) theoretically examined surface waves in a rectangular container with a square base due to its resonant linear oscillations in various horizontal directions. Under the assumption of infinite fluid depth, they derived nonlinear evolution equations for the complex amplitudes of two degenerate modes excited by the oscillations. By solving these equations numerically, they obtained response curves of two kinds of stationary solutions expressing regular rotating and non-rotating waves, and also Poincaré sections of non-stationary solutions expressing periodic or chaotic amplitude modulations, for several values of parameters such as the direction of oscillation and the coefficient of linear damping. Ikeda et al (2012) also theoretically investigated a similar problem but of a rectangular base and a finite fluid depth by deriving equations of motion for two dominant modes and five higher modes. 
By solving these equations, they examined the dependences of response curves of two kinds of stationary solutions on the direction of oscillation and on the aspect ratio of the rectangular base of the container, and also found non-stationary solutions expressing chaotic or periodic amplitude modulations. Moreover, they performed experiments on this problem with a container of square base for three directions of oscillation. They observed excitation of surface waves of constant or modulated amplitudes, and showed that the dependence of amplitudes of these waves at two measurement points on slowly increasing or decreasing forcing frequency agrees well with the results of the above theoretical study.

In the present study, surface waves due to the resonant horizontal elliptic motion (including linear motion as its special case) of a rectangular container with a square base are investigated for a fluid of finite depth, in which the frequency of elliptic motion is assumed to be close to the natural frequency of two degenerate modes with the smallest natural frequency. The main purpose of this study is to examine the dependence of excited surface waves on the parameters characterizing this elliptic motion with a weakly nonlinear theory, because the waves due to this motion have not been previously examined before. Moreover, in the special case of linear motion of the container, we examine excited surface waves for various fluid depths and compare with experimental results in a preceding study.

In section 2, using the reductive perturbation method and including a linear damping effect, we derive nonlinear evolution equations for complex amplitudes of the two degenerate modes. In section 3, numerical results on response curves of stationary solutions to these equations, expressing regular rotating or non-rotating waves, and on the Poincaré sections of non-stationary solutions, displaying chaotic or periodic amplitude modulations of excited waves, are shown. Moreover, our results on stationary or non-stationary solutions for a linear motion of the container are compared with experimental results obtained by Ikeda et al (2012) in section 4. Finally, section 5 is devoted to conclusions.

\section{Formulation}

\subsection{Derivation of nonlinear evolution equations}

We consider three-dimensional surface waves in a rectangular container of square base with side length $\pi L$ due to its resonant horizontal elliptic or linear motions. In the following part, all variables are nondimensionalized by the length $L$ and the time $\sqrt{L / g}$, where $g$ is the gravitational acceleration. We first introduce the Cartesian coordinates $(\tilde{x}, \tilde{y}, \tilde{z})$ of an inertial system, where $\tilde{x}$ and $\tilde{y}$ are horizontal coordinates. We assume that the horizontal displacement of the container is expressed as

$$
\left\{\begin{array}{l}
\tilde{x}=-a_{0}(\cos \phi \cos \theta \cos \Omega t-\sin \phi \sin \theta \sin \Omega t), \\
\tilde{y}=-a_{0}(\cos \phi \sin \theta \cos \Omega t+\sin \phi \cos \theta \sin \Omega t),
\end{array}\right.
$$




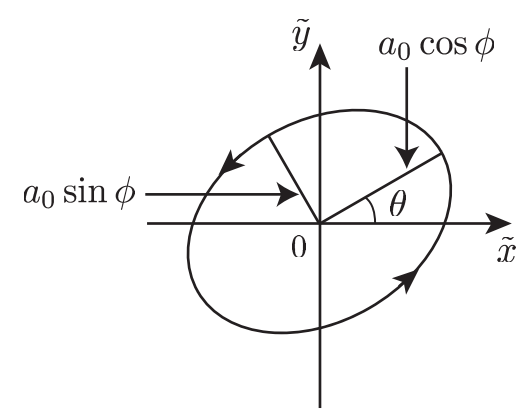

Figure 1. Orbit of container's motion.

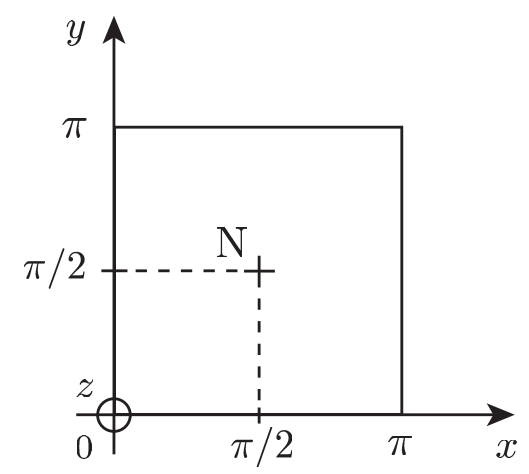

Figure 2. Cartesian coordinates fixed to a container.

where $t$ is the time, $a_{0}$ is a positive constant, and $\theta$ and $\phi$ are constants satisfying $0^{\circ} \leq \theta \leq 45^{\circ}$ and $0^{\circ} \leq \phi \leq 45^{\circ}$. Therefore, the container moves with frequency $\Omega$, and the orbit of its motion is elliptic, as illustrated in figure 1 . Here $\theta$ is the angle between the major axis of the elliptic orbit and the $\tilde{x}$-axis. Since the lengths of the major and minor axes of the elliptic orbit are expressed as $2 a_{0} \cos \phi$ and $2 a_{0} \sin \phi$, respectively, the ratio of the length of the minor axis to the length of the major axis is $\tan \phi$. Next, we introduce Cartesian coordinates $(x, y, z)$ fixed to the container, as illustrated in figure 2 . Here, $x$ and $y$ are horizontal coordinates along the sidewalls of the container in which sidewalls are expressed as $x=0, \pi$ and $y=0, \pi$, whereas $z$ is the upward vertical coordinate in which a quiescent surface is defined by $z=0$.

We assume irrotational flow of an incompressible inviscid fluid. Therefore, using a velocity potential $\Phi(x, y, z, t)$, the $(x, y, z)$ components of fluid velocity $\boldsymbol{u}$ are expressed as $\boldsymbol{u}=\nabla \Phi$. The displacement of the free surface is expressed by $z=\eta(x, y, t)$. The equation of continuity is written as

$$
\operatorname{div} \boldsymbol{u}=0 .
$$

Using the velocity potential $\Phi$, this equation is expressed as

$$
\nabla^{2} \Phi=0, \quad \text { for }-h \leq z \leq \eta,
$$

where $\nabla^{2}$ is the Laplacian, and $h$ is a dimensionless depth obtained by dividing a fluid depth by L. From Bernoulli's theorem and (1), the dynamical condition at the free 
surface is given as

$$
\begin{aligned}
\partial_{t} \Phi & +\eta+f(\cos \phi \cos \theta \cos \Omega t-\sin \phi \sin \theta \sin \Omega t) x \\
& +f(\cos \phi \sin \theta \cos \Omega t+\sin \phi \cos \theta \sin \Omega t) y+\frac{1}{2}(\nabla \Phi)^{2}=0, \quad \text { at } z=\eta,
\end{aligned}
$$

where $f=a_{0} \Omega^{2}$. The kinematic condition at the free surface is written as

$$
\partial_{t} \eta-\partial_{z} \Phi+\left(\partial_{x} \eta\right)\left(\partial_{x} \Phi\right)+\left(\partial_{y} \eta\right)\left(\partial_{y} \Phi\right)=0, \quad \text { at } z=\eta .
$$

Moreover, boundary conditions at the sidewalls and bottom of the container are given by

$$
\begin{cases}\partial_{x} \Phi=0, & \text { at } x=0, \pi, \\ \partial_{y} \Phi=0, & \text { at } y=0, \pi, \\ \partial_{z} \Phi=0, & \text { at } z=-h .\end{cases}
$$

From (2) and boundary condition (5), the following velocity potential of the $(m, n)$ surface-wave eigenmode is obtained:

$$
\Phi=T(t) \cos (m x) \cos (n y) \frac{\cosh \left[\lambda_{m, n}(z+h)\right]}{\cosh \left(\lambda_{m, n} h\right)}
$$

where $m$ and $n$ are non-negative integers satisfying $(m, n) \neq(0,0)$, and $\lambda_{m, n}=$ $\sqrt{m^{2}+n^{2}}$. Moreover, the dispersion relation for the natural frequency $\omega_{m, n}$ of $(m, n)$ mode,

$$
\omega_{m, n}=\sqrt{\lambda_{m, n} \tanh \left(\lambda_{m, n} h\right)}
$$

is derived from the linearized version of (3) and (4) when terms due to the motion of the container are neglected. Using this $\omega_{m, n}, T(t)$ in $(6)$ is written as

$$
T(t)=c \mathrm{e}^{\mathrm{i} \omega_{m, n} t}+\text { c.c. }
$$

where c.c. denotes the complex conjugate of the preceding terms, and $c$ is a constant. In the present study, we consider the case of resonant motion of the container in which the frequency $\Omega$ of this motion is close to the natural frequency of the $(1,0)$ and $(0,1)$ modes, $\omega_{1,0}=\omega_{0,1}=\sqrt{\tanh h}$.

In the following part of this subsection, we derive nonlinear equations that govern the time evolution of surface waves excited by this resonant motion with the reductive perturbation method. Expanding $\Phi$ and its partial derivatives at $z=\eta$ in (3) and (4) around $z=0$, and neglecting the terms of fourth or higher order with respect to $\eta$ and $\Phi$, we obtain

$$
\begin{aligned}
\partial_{t} \Phi & +\eta+f(\cos \phi \cos \theta \cos \Omega t-\sin \phi \sin \theta \sin \Omega t) x \\
& +f(\cos \phi \sin \theta \cos \Omega t+\sin \phi \cos \theta \sin \Omega t) y+\eta \partial_{z} \partial_{t} \Phi \\
& +\frac{1}{2}(\nabla \Phi)^{2}+\frac{1}{2} \eta^{2} \partial_{z}^{2} \partial_{t} \Phi+\frac{1}{2} \eta \partial_{z}\left[(\nabla \Phi)^{2}\right]=0, \quad \text { at } z=0,
\end{aligned}
$$

and

$$
\begin{aligned}
\partial_{t} \eta & -\partial_{z} \Phi-\eta \partial_{z}^{2} \Phi+\left(\partial_{x} \eta\right)\left(\partial_{x} \Phi\right)+\left(\partial_{y} \eta\right)\left(\partial_{y} \Phi\right) \\
& -\frac{1}{2} \eta^{2} \partial_{z}^{3} \Phi+\eta\left(\partial_{x} \eta\right)\left(\partial_{z} \partial_{x} \Phi\right)+\eta\left(\partial_{y} \eta\right)\left(\partial_{z} \partial_{y} \Phi\right)=0, \quad \text { at } z=0 .
\end{aligned}
$$


Now we assume that

$$
f=\epsilon^{3} \pi
$$

where $\epsilon>0$ satisfies $\epsilon \ll 1$. We then expand $\Phi$ and $\eta$ as

$$
\left\{\begin{array}{l}
\Phi=\epsilon \Phi_{1}+\epsilon^{2} \Phi_{2}+\epsilon^{3} \Phi_{3}+\cdots \\
\eta=\epsilon \eta_{1}+\epsilon^{2} \eta_{2}+\epsilon^{3} \eta_{3}+\cdots .
\end{array}\right.
$$

We also assume that $\Omega-H=O\left(\epsilon^{2}\right)$, where $H=\sqrt{\tanh h}$, and write $\Omega$ as

$$
\Omega=H+\epsilon^{2} \delta,
$$

with the detuning parameter $\delta$. Moreover, in order to describe the slow time evolution of amplitudes and phases of excited surface waves, we introduce the slow time variable $\tau=\epsilon^{2} t$, and then assume that $\Phi$ and $\eta$ depend on not only $t$ but also $\tau$. Therefore, the operator $\partial_{t}$ in $(7)$ and (8) is replaced by $\partial_{t}+\epsilon^{2} \partial_{\tau}$. The above scaling is chosen so that the lowest-order effect of nonlinearity, which is of $O\left(\epsilon^{3}\right)$ if the amplitude of excited surface waves is of $O(\epsilon)$, is of the same order as the effects of forcing and detuning.

Under the above assumptions, we obtain

$$
\begin{cases}\partial_{t} \Phi_{1}+\eta_{1}=0, & \text { at } z=0, \\ \partial_{t} \eta_{1}-\partial_{z} \Phi_{1}=0, & \text { at } z=0,\end{cases}
$$

from the terms of $O(\epsilon)$ in (7) and (8). Equations obtained in $O\left(\epsilon^{2}\right)$ and $O\left(\epsilon^{3}\right)$ of (7) and (8) are shown in Appendix A as (A.1) and (A.2), respectively. Moreover, at $O\left(\epsilon^{j}\right)(j=1,2, \ldots)$ of $(2)$ and $(5)$, the following equations for $\Phi_{j}$ are obtained:

$$
\left\{\begin{array}{ll}
\nabla^{2} \Phi_{j}=0, & \text { for }-h \leq z \leq 0, \\
\partial_{x} \Phi_{j}=0, & \text { at } x=0, \pi, \\
\partial_{y} \Phi_{j}=0, & \text { at } y=0, \pi, \\
\partial_{z} \Phi_{j}=0, & \text { at } z=-h,
\end{array} \quad(j=1,2, \ldots) .\right.
$$

Equations obtained at $O(\epsilon)$, that is (12) and (13) with $j=1$ for $\Phi_{1}$ and $\eta_{1}$, are the same as the linearized version of the governing equations $(2)-(5)$ of surface waves with forcing terms neglected. Under assumption (11), only the $(1,0)$ and $(0,1)$ modes of natural frequency $H$ are expected to be resonantly excited. Therefore, $\Phi_{1}$ and $\eta_{1}$ are determined as

$$
\left\{\begin{array}{l}
\Phi_{1}=[a(\tau) \cos x+b(\tau) \cos y] \mathrm{e}^{\mathrm{i} H t} \frac{\cosh (z+h)}{\cosh h}+\text { c.c. } \\
\eta_{1}=-\mathrm{i} H[a(\tau) \cos x+b(\tau) \cos y] \mathrm{e}^{\mathrm{i} H t}+\text { c.c. }
\end{array}\right.
$$

where $a(\tau)$ and $b(\tau)$ are functions of $\tau$, and $-\mathrm{i} H a(\tau)$ and $-\mathrm{i} H b(\tau)$ express the complex amplitudes of the $(1,0)$ and $(0,1)$ modes, respectively. Substituting (14) into (A.1) and using (13) with $j=2$, we obtain the equations for $\Phi_{2}$ and $\eta_{2}$. By solving these equations, we find that $\Phi_{2}$ and $\eta_{2}$ are given as (A.3) in Appendix A.

If $\Phi_{1}, \eta_{1}, \Phi_{2}$ and $\eta_{2}$ given in (14) and (A.3) are substituted into (A.2), two equations for $\Phi_{3}$ and $\eta_{3}$ are obtained. When these equations multiplied by $\cos x$ are integrated over 
a square region defined by $0 \leq x \leq \pi$ and $0 \leq y \leq \pi$, a pair of equations are obtained. From the solvability condition of these equations, a nonlinear time evolution equation for $a$ and $b$ is derived. An additional time evolution equation for $a$ and $b$ is also derived from the solvability condition of a pair of equations resulting from the integration of the two equations for $\Phi_{3}$ and $\eta_{3}$ multiplied by $\cos y$ over the same region. Moreover, if we include the effect of linear damping, we finally obtain the following nonlinear time evolution equation:

$$
\left\{\begin{array}{l}
\frac{\mathrm{d} A}{\mathrm{~d} \tau}=-\alpha A-\mathrm{i} \delta A-\mathrm{i} R_{1}|A|^{2} A-\mathrm{i} R_{2}|B|^{2} A-\mathrm{i} R_{3} A^{*} B^{2}+\cos \phi \cos \theta+\mathrm{i} \sin \phi \sin \theta \\
\frac{\mathrm{d} B}{\mathrm{~d} \tau}=-\alpha B-\mathrm{i} \delta B-\mathrm{i} R_{1}|B|^{2} B-\mathrm{i} R_{2} B|A|^{2}-\mathrm{i} R_{3} A^{2} B^{*}+\cos \phi \sin \theta-\mathrm{i} \sin \phi \cos \theta
\end{array}\right.
$$

where $A(\tau)=a(\tau) \mathrm{e}^{-\mathrm{i} \delta \tau}$ and $B(\tau)=b(\tau) \mathrm{e}^{-\mathrm{i} \delta \tau}, \alpha$ is the coefficient of linear damping, and $*$ denotes complex conjugate. $R_{1}, R_{2}$ and $R_{3}$ are constants depending only on $h$, defined by

$$
\left\{\begin{array}{l}
R_{1}=\frac{1}{16 H^{5}}\left(2 H^{12}+3 H^{8}+12 H^{4}-9\right), \\
R_{2}=\frac{1}{2}\left[-H^{3}+S_{2}\left(1-\frac{\sqrt{2}}{2} H^{2} \tanh (\sqrt{2} h)\right)-\frac{1}{2} H^{3} S_{4}+\frac{1}{2} H^{7}\right], \\
R_{3}=\frac{H^{3}}{2}\left(1+\frac{1}{2} H^{4}\right),
\end{array}\right.
$$

where $S_{2}$ and $S_{4}$ are given in (A.4) of Appendix A. Equation (15) contains $\phi$ and $\theta$ in addition to $\alpha, \delta$ and $h$ as parameters. In the limit of $h \rightarrow \infty$ with $\phi$ fixed to $0^{\circ},(15)$ reduces to the nonlinear time evolution equations derived by Yoshimatsu and Funakoshi (2001). Moreover, the nonlinear coefficients $R_{1}, R_{2}$ and $R_{3}$ in (15) are the same as those in the model equations for Faraday waves in a square container with a finite depth derived by Nagata (1989).

\subsection{Rotation of wave pattern}

To characterize the energy of excited surface wave, we introduce a variable $E$ defined by $E=\frac{H^{2}}{2}\left(|A|^{2}+|B|^{2}\right)$. Because $E$ is equal to the potential energy and also equal to the kinematic energy of the excited surface waves averaged over one period at the lowest order of $\epsilon, \sqrt{E}$ is a measure of amplitude of these waves.

We also introduce a real variable $M=\frac{\mathrm{i}}{2} H^{2}\left(A^{*} B-A B^{*}\right)=\frac{\mathrm{i}}{2} H^{2}\left(a^{*} b-a b^{*}\right)$ to characterize the rotation of the wave pattern. If only the $(1,0)$ and $(0,1)$ modes of surface waves are excited, there is a nodal point $\mathrm{N}$ at $(x, y)=\left(\frac{\pi}{2}, \frac{\pi}{2}\right)$ (see figure 2 ). Using the polar coordinates $(r, \psi)$ around $\mathrm{N}$ defined by

$$
\left\{\begin{array}{l}
x=r \cos \psi+\frac{\pi}{2}, \\
y=r \sin \psi+\frac{\pi}{2}
\end{array}\right.
$$



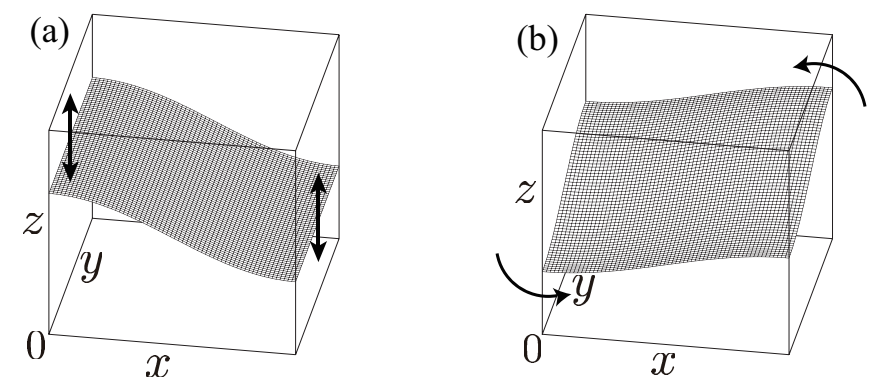

Figure 3. Schematic diagrams of excited surface waves. (a) Surface waves with $M=0$ (and $B=0$ ). (b) Surface waves with $M>0$.

$\eta_{1}$ given by (14) is approximately expressed as

$$
\eta_{1}=-2 H r\left[f_{a}(t) \cos \psi+f_{b}(t) \sin \psi\right],
$$

for small $r$, where

$$
\left\{\begin{array}{l}
f_{a}(t)=a_{r} \sin H t+a_{i} \cos H t, \\
f_{b}(t)=b_{r} \sin H t+b_{i} \cos H t,
\end{array}\right.
$$

and $a_{r}=\operatorname{Re}[a], a_{i}=\operatorname{Im}[a], b_{r}=\operatorname{Re}[b]$ and $b_{i}=\operatorname{Im}[b]$. If $\psi_{n}(t)$ is defined as the value of $\psi$ for which this $\eta_{1}$ is zero for non-zero $r$ at the time $t$, we obtain

$$
\frac{\mathrm{d} \psi_{n}}{\mathrm{~d} t}=\frac{M}{H\left(f_{a}^{2}+f_{b}^{2}\right)} .
$$

Therefore, because the nodal line satisfying $\eta_{1}=0$ near $\mathrm{N}$ rotates around $\mathrm{N}$ at the angular velocity (17), we can say that the pattern of excited waves rotates counterclockwise (clockwise) if $M>0(M<0)$. Moreover, if $M \neq 0$, the nodal line rotates once in one period $\frac{2 \pi}{H}$ of $\eta_{1}$, but with time-dependent angular velocity (17), because $f_{a}$ and $f_{b}$ depend on $t$. From the definitions of $M$ and $E$, the relation $\left|\frac{M}{E}\right| \leq 1$ is derived. If $\left|\frac{M}{E}\right|=1$ is satisfied, angular velocity (17) is found to be $\pm H$ for all $t$, which implies uniform rotation of the wave pattern. Therefore, it is expected that the rotation of the wave pattern is easily recognized if $\left|\frac{M}{E}\right|$ is close to 1 . However, if $\left|\frac{M}{E}\right|$ is close to 0 , angular velocity (17) is small for most $t$, whereas it takes a large absolute value for $t$ within small ranges that are observed twice in one period, implying a highly non-uniform rotation. Therefore, we can say that the time evolution of the wave pattern for small but non-zero $\left|\frac{M}{E}\right|$ is similar to a planar oscillation rather than a rotation.

It can be also shown that the point of maximum $\eta_{1}$ visits four corners of the container in a counterclockwise (clockwise) order if $M>0(M<0)$, as was explained by Yoshimatsu and Funakoshi (2001). Moreover, when $M=0$ is satisfied, the maximum of $\eta_{1}$ is observed alternately at two opposite corners of the container (for $A= \pm B$ ), or observed alternately on its opposite sidewalls (for $A=0$ or $B=0$ ). Schematic diagrams of excited surface waves with $M=0$ and $M>0$ are shown in figure 3 . 


\section{Numerical results}

\subsection{Stationary solutions}

Stationary solutions of (15), which correspond to regular surface waves of frequency $\Omega$ with time-independent amplitude, are calculated numerically by the Brent method for $\phi$ and $\theta$ within the regions of $0^{\circ} \leq \theta \leq 45^{\circ}$ and $0^{\circ} \leq \phi \leq 45^{\circ}$. Moreover, their stability is examined on the basis of eigenvalues of the Jacobian matrix of (15). Equation (15) has stationary solutions with $M=0$, called non-rotating waves, only when $\phi=0^{\circ}$ and $\theta=0^{\circ}$ or $45^{\circ}$. The stationary solutions of non-rotating waves satisfy $B=0$ if $\phi=\theta=0^{\circ}$, whereas they satisfy $A=B$ when $\theta=45^{\circ}$ and $\phi=0^{\circ}$. Moreover, (15) has also stationary solutions with $M \neq 0$ called rotating waves. For $\phi>0^{\circ}$, stationary solutions with $M>0$ that correspond to waves rotating in the same direction as that of the container's elliptic motion are called co-rotating waves, whereas stationary solutions with $M<0$ expressing the rotation of waves in the opposite direction to that of the container's motion are called counter-rotating waves.

\subsubsection{Dependence on $\theta$ and $\phi$}

In the examination of dependence of stationary solutions on $\theta$ and $\phi$, the coefficient of linear damping $\alpha$ is fixed to 0.3. Moreover, $h$ is fixed to $\pi$, which yields $R_{1}=0.493$, $R_{2}=-0.0452$ and $R_{3}=0.744$ in (15).

Figure 4 shows response curves expressing the dependence of $\sqrt{E}$ of stationary solutions on $\delta$ for a few $\phi$ with $\theta$ fixed to $0^{\circ}$. For $\phi=0^{\circ}$, response curves shown in figure 4(a) are similar to those obtained by Yoshimatsu and Funakoshi(2001) for $h=\infty$. That is, in addition to a branch of non-rotating waves that extends to $\delta \rightarrow \pm \infty$, there is a bounded branch of rotating waves. The latter branch shows a pair of solutions with $M>0$ and $M<0$ that give the same value of $\sqrt{E}$ owing to the symmetry of system. Moreover, this branch emerges through pitchfork bifurcations at $\mathrm{P}_{1}$ and $\mathrm{P}_{2}$ on the branch of non-rotating waves of figure 4 (a). For $\delta$ satisfying $-4<\delta<1$, we observe both rotating and non-rotating waves of large amplitude. However, if $|\delta|$ is sufficiently large, only non-rotating waves of small amplitude exist. Non-rotating waves are stable for large $|\delta|$, but are unstable for $\delta$ between $\mathrm{P}_{2}$ and $\mathrm{S}$, the saddle-node bifurcation point on the branch of non-rotating waves shown in figure 4(a). There are two regions of $\delta$ where rotating waves are stable. They are delimited by the saddle-node bifurcation points on the branch of these waves, and by the points $\mathrm{H}_{1}$ and $\mathrm{H}_{2}$ of Hopf bifurcations shown in figure 4(a). There are no stable stationary solutions for $\delta$ between $\mathrm{S}$ and $\mathrm{H}_{2}$.

For $\phi>0^{\circ}$, there are no stationary solutions of non-rotating waves. As $\phi$ increases from $0^{\circ}$, the branches of non-rotating and rotating waves for $\phi=0^{\circ}$ change to two branches of rotating waves composed of an unbounded branch $\mathrm{C}_{\mathrm{ub}}$ extending to $\delta \rightarrow \pm \infty$ and a bounded branch $\mathrm{C}_{\mathrm{b}}$ of a closed curve observed in a finite range of $\delta$, as illustrated in figures $4(\mathrm{~b})$ and (c). Here it is noted that since solutions of co-rotating and counterrotating waves give different values of $\sqrt{E}$ for $\phi>0^{\circ}$, a degenerate response curve of rotating waves for $\phi=0^{\circ}$ splits into two curves for small but non-zero $\phi$. It is found 

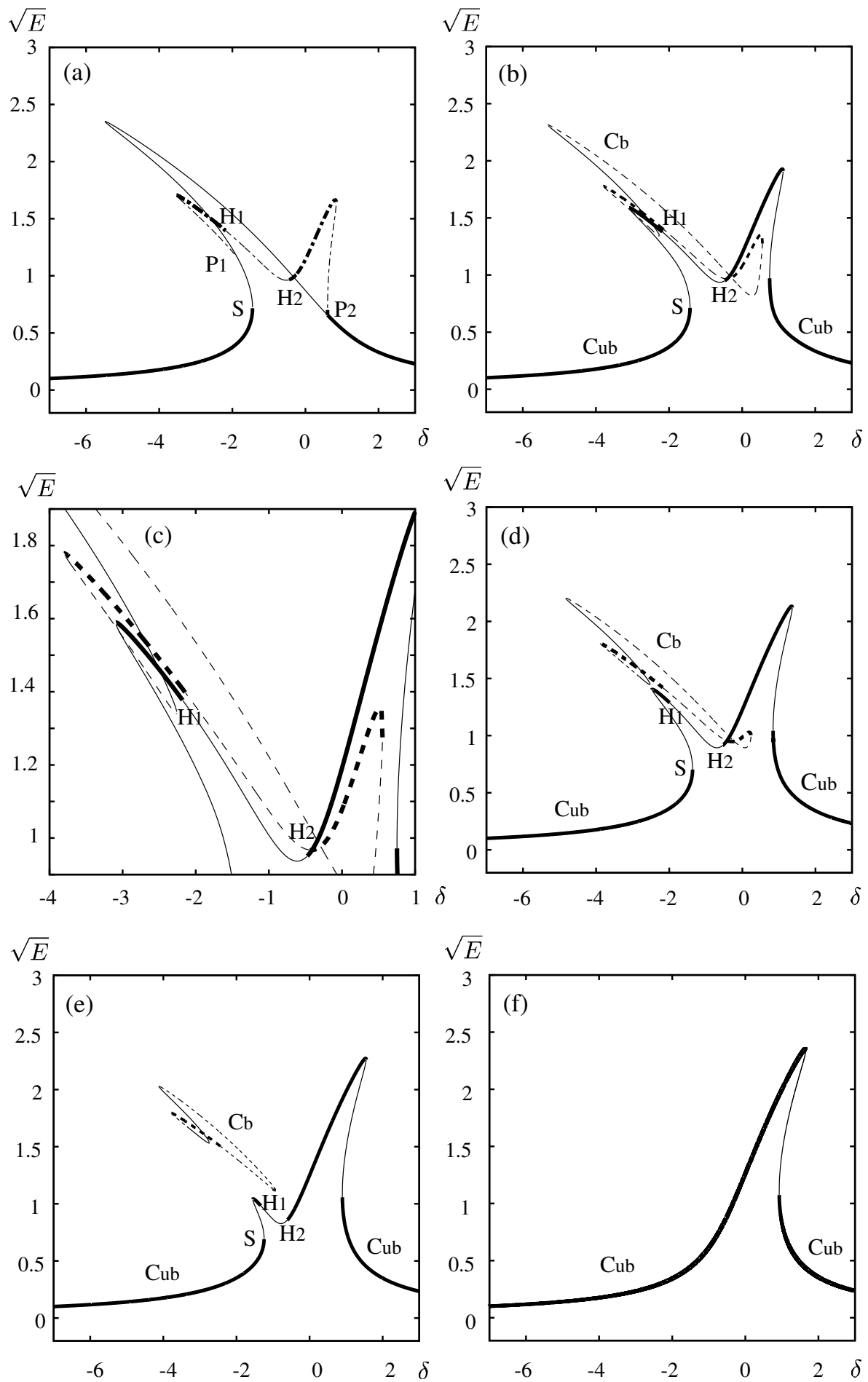

Figure 4. Response curves for $\alpha=0.3, h=\pi$ and $\theta=0^{\circ}$. (a) $\phi=0^{\circ}$, (b), (c) $\phi=10^{\circ}$, (d) $\phi=20^{\circ}$, (e) $\phi=30^{\circ}$ and (f) $\phi=45^{\circ}$. (c) is a magnification of (b). Solid lines are stationary solutions with $M=0$ in (a), and with $M>0$ in (b) (f). Dotted broken lines are stationary solutions with $M \neq 0$ in (a), and broken lines are those with $M<0$ in (b) - (e). Thick and thin lines denote stable and unstable solutions, respectively. $\mathrm{C}_{\mathrm{ub}}$ and $\mathrm{C}_{\mathrm{b}}$ are unbounded and bounded branches of response curves, respectively. $\mathrm{S}, \mathrm{H}_{j}(j=1,2)$ and $\mathrm{P}_{j}(j=1,2)$ denote the points of saddle-node bifurcation, Hopf bifurcation and pitchfork bifurcation, respectively. 
for positive $\phi$ that only co-rotating waves are observed on $\mathrm{C}_{\mathrm{ub}}$, but that there are both co-rotating and counter-rotating waves on $\mathrm{C}_{\mathrm{b}}$, as shown in figures $4(\mathrm{~b})-(\mathrm{e})$.

For small $\phi$, there are two large $\sqrt{E}$ portions of the $\mathrm{C}_{\mathrm{ub}}$ curve for positive and negative $\delta$. With the increase in $\phi$ to $45^{\circ}$, one of these portions for positive $\delta$ grows slightly, but the other portion for negative $\delta$ shrinks and disappears, as shown in figures $4(\mathrm{~b})-(\mathrm{f})$. For $\phi$ where both of the two large $\sqrt{E}$ portions are observed, we find on $\mathrm{C}_{\mathrm{ub}}$ two Hopf bifurcations at $\mathrm{H}_{1}$ and $\mathrm{H}_{2}$ between the two large $\sqrt{E}$ portions. Corotating waves on $\mathrm{C}_{\mathrm{ub}}$ are stable within two regions of $\delta$ delimited by $\mathrm{H}_{1}\left(\mathrm{H}_{2}\right)$ and the saddle-node bifurcation point in the large $\sqrt{E}$ portion for negative (positive) $\delta$, and are unstable for $\delta$ between $\mathrm{H}_{1}$ and $\mathrm{H}_{2}$. With increasing $\phi, \mathrm{H}_{1}$ and $\mathrm{H}_{2}$ come closer to each other and disappear at $\phi=33.78^{\circ}$ after their collision. Therefore, for $\phi$ sufficiently close to $45^{\circ}$, one or two stable co-rotating waves are observed on $\mathrm{C}_{\mathrm{ub}}$ for all $\delta$, as illustrated in figure $4(\mathrm{f})$.

For small non-zero $\phi$, we find on $\mathrm{C}_{\mathrm{b}}$ two regions of $\delta$ where stable counter-rotating waves are observed, which are bounded by the points of Hopf bifurcations and saddlenode bifurcations on $\mathrm{C}_{\mathrm{b}}$, as shown in figures $4(\mathrm{~b})-(\mathrm{d})$. However, co-rotating waves on $\mathrm{C}_{\mathrm{b}}$ are always unstable. As $\phi$ increases to $45^{\circ}$, we observe the tendency that stable counterrotating waves are found in smaller regions of $\delta$ owing to the shrinking of $\mathrm{C}_{\mathrm{b}}$. Because $\mathrm{C}_{\mathrm{b}}$ vanishes at $\phi=43.96^{\circ}$, stable counter-rotating waves exist only for $\phi \leq 43.96^{\circ}$.

For $\phi=45^{\circ}$ that corresponds to a circular motion of the container, only co-rotating waves on $\mathrm{C}_{\mathrm{ub}}$ are observed for all $\delta$, as shown in figure $4(\mathrm{f})$. There are two saddle-node bifurcations on $\mathrm{C}_{\mathrm{ub}}$, and two stable co-rotating waves exist for $\delta$ between the points of these bifurcations.

Some of the solutions of rotating waves shown above are associated with small values of $\left|\frac{M}{E}\right|$. That is, for all $\phi$ satisfying $0^{\circ} \leq \phi \leq 45^{\circ}$, although $\left|\frac{M}{E}\right|$ for stable rotating waves is larger than 0.5 if $\delta$ satisfies $-0.5<\delta<1,\left|\frac{M}{E}\right|$ for stable rotating waves of large $\sqrt{E}$ is smaller than 0.2 for $-4<\delta<-2$. Therefore, in the latter case, it may be difficult to recognize the rotation of the wave pattern in experiments even if $M$ is not zero.

Response curves for $\theta$ fixed to $22.5^{\circ}$ and $45^{\circ}$ are shown in figure 5. Response curves for $\theta=45^{\circ}$ and $\phi=0^{\circ}$, shown in figure 5(a), are similar to those obtained by Yoshimatsu and Funakoshi (2001) for $h=\infty$. That is, there is a branch of non-rotating waves that extends to $\delta \rightarrow \pm \infty$ as well as a branch displaying a pair of rotating waves with $M>0$ and $M<0$ that emerges from the branch of non-rotating waves through pitchfork bifurcations at $\mathrm{P}_{1}$ and $\mathrm{P}_{2}$ of figure 5(a). Unlike the case of $\theta=\phi=0^{\circ}, \mathrm{P}_{1}$ is located between the saddle-node bifurcation point of large $\sqrt{E}$ and $\mathrm{P}_{2}$ on the branch of non-rotating waves, which can cause a large region of $\delta$ where non-rotating waves are stable.

If $\phi$ slightly increases from $0^{\circ}$ with $\theta$ fixed to $45^{\circ}$, response curves are composed of an unbounded branch $\mathrm{C}_{\mathrm{ub}}$ and a bounded branch $\mathrm{C}_{\mathrm{b}}$ of rotating waves similarly to the case of $\theta=0^{\circ}$, as illustrated in figure $5(\mathrm{~b})$ for $\phi=20^{\circ}$. However, for most $\phi$ both co-rotating and counter-rotating waves are observed as stable solutions on $\mathrm{C}_{\mathrm{ub}}$ 

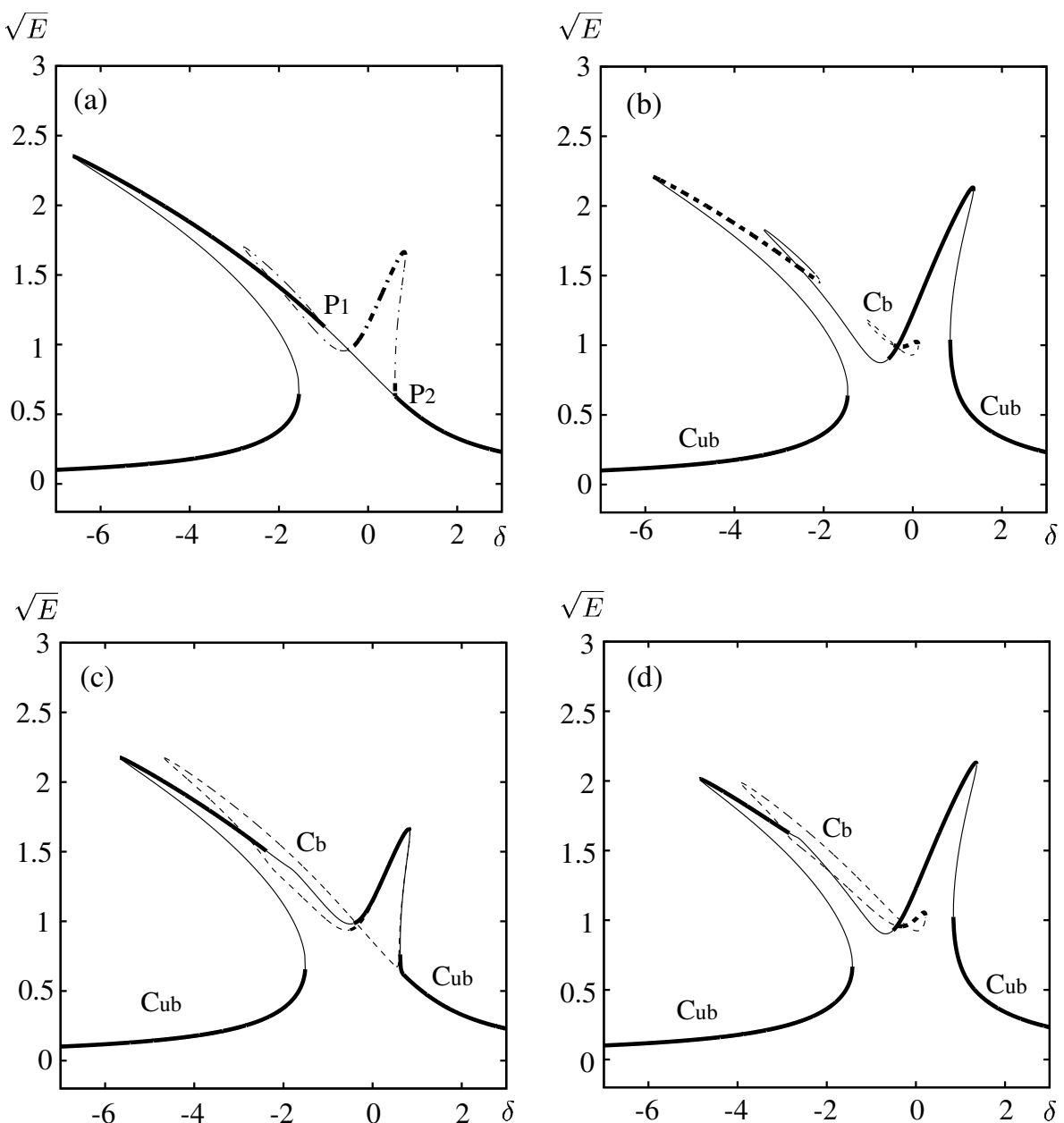

Figure 5. Response curves for $\alpha=0.3$ and $h=\pi$. (a) $\theta=45^{\circ}$ and $\phi=0^{\circ}$, (b) $\theta=45^{\circ}$ and $\phi=20^{\circ}$, (c) $\theta=22.5^{\circ}$ and $\phi=0^{\circ}$, (d) $\theta=22.5^{\circ}$ and $\phi=20^{\circ}$. Solid lines are stationary solutions with $M=0$ in (a), and with $M>0$ in (b) - (d). Dotted broken lines are stationary solutions with $M \neq 0$ in (a), and broken lines are those with $M<0$ in (b) - (d). Thick and thin lines denote stable and unstable solutions, respectively. $\mathrm{C}_{\mathrm{ub}}$ and $\mathrm{C}_{\mathrm{b}}$ are unbounded and bounded branches of response curves, respectively. $\mathrm{P}_{1}$ and $\mathrm{P}_{2}$ denote pitchfork bifurcation points.

unlike the case of $\theta=0^{\circ}$. Since the branch $\mathrm{C}_{\mathrm{b}}$ shrinks as $\phi$ increases from $0^{\circ}$ and disappears at $\phi=22.52^{\circ}$, stable counter-rotating waves on $\mathrm{C}_{\mathrm{b}}$ are observed only for $\phi \leq 22.52^{\circ}$. Moreover, the region of $\delta$ where no stable co-rotating waves are observed, which is $-1.457<\delta<-0.553$ for $\phi=20^{\circ}$, also becomes narrower with the increase in $\phi$. Since this region disappears at $\phi=36.87^{\circ}$, stable co-rotating waves are found for all $\delta$ if $\phi \geq 36.87^{\circ}$. The $\delta$ region of stable counter-rotating waves on $\mathrm{C}_{\mathrm{ub}}$, which is $-5.674<\delta<-2.081$ for $\phi=20^{\circ}$ (shown in figure $5(\mathrm{~b})$ ), shrinks with the increase in $\phi$ and vanishes at $\phi=43.96^{\circ}$, beyond which no stable counter-rotating waves exist for any $\delta$.

For $\theta$ different from $0^{\circ}$ or $45^{\circ}$, solutions of non-rotating waves do not exist even if $\phi=0^{\circ}$. Therefore, response curves for $0^{\circ}<\theta<45^{\circ}$ are composed of an unbounded 


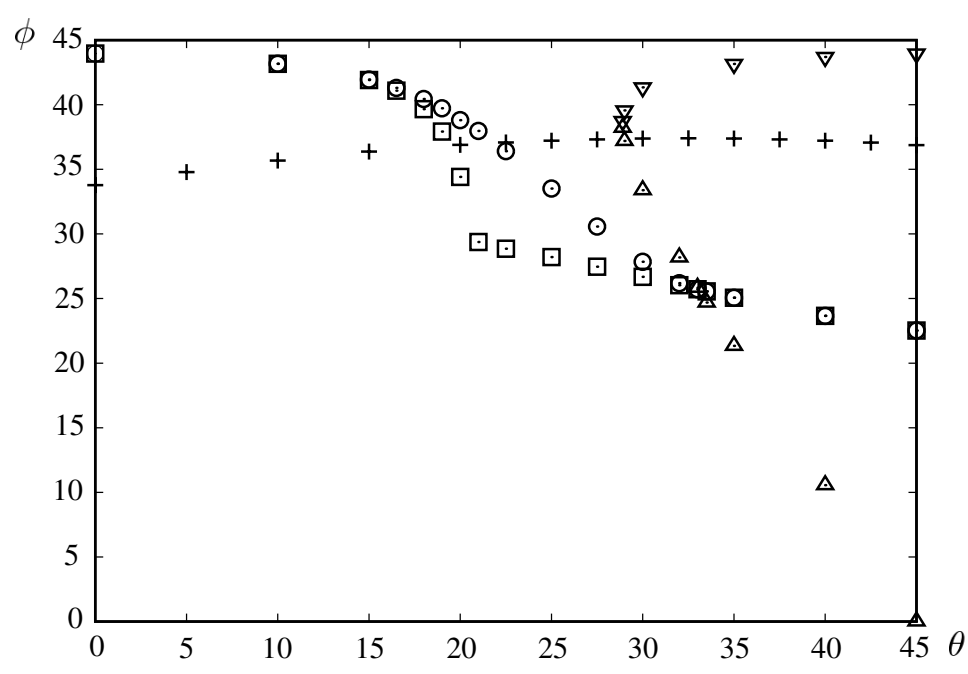

Figure 6. The boundaries of $(\theta, \phi)$ regions where stable or unstable counter-rotating waves exist on $\mathrm{C}_{\mathrm{ub}}$ or $\mathrm{C}_{\mathrm{b}}$, and $(\theta, \phi)$ region where stable co-rotating waves are observed on $\mathrm{C}_{\mathrm{ub}}$ for all $\delta . \mathrm{O}, \square, \Delta, \nabla$ and + denote the values of $\phi_{\mathrm{b}}^{\text {all }}, \phi_{\mathrm{b}}^{\text {stable }}, \phi_{\mathrm{ub}}^{\min }, \phi_{\mathrm{ub}}^{\max }$ and $\phi_{\mathrm{ub}}^{+}$, respectively, for several $\theta . \alpha=0.3$ and $h=\pi$.

branch $\mathrm{C}_{\mathrm{ub}}$ and a bounded branch $\mathrm{C}_{\mathrm{b}}$ of rotating waves for any $\phi$, as illustrated in figures $5(\mathrm{c})$ and (d) for $\theta=22.5^{\circ}$ and $\phi=0^{\circ}$ or $20^{\circ}$. It is noted that near the large $\sqrt{E}$ portion for positive $\delta$ in figure $5(\mathrm{c})$, there are both solutions with $M>0$ on $\mathrm{C}_{\mathrm{ub}}$ and solutions with $M<0$ on $\mathrm{C}_{\mathrm{b}}$, although it is difficult to recognize them because they give almost the same values of $\sqrt{E}$. As illustrated in figures $5(\mathrm{c})$ and (d), for $\theta=22.5^{\circ}$, only rotating waves with $M<0$ are observed on $\mathrm{C}_{\mathrm{b}}$ but only those with $M>0$ exist on $\mathrm{C}_{\mathrm{ub}}$ for any $\phi$, unlike the case of $\theta=45^{\circ}$ and $\phi>0^{\circ}$. Moreover, as $\phi$ increases from $0^{\circ}$, stable counter-rotating waves on $\mathrm{C}_{\mathrm{b}}$, which are observed in figure $5(\mathrm{~d})$ for $\phi=20^{\circ}$ in a small region of $\delta$ around zero, disappear at $\phi=28.86^{\circ}$. Furthermore, stable co-rotating waves are found to exist for all $\delta$ if $\phi$ is larger than $37.08^{\circ}$.

Response curves are examined for several $\theta$ and $\phi$ satisfying $0^{\circ} \leq \theta \leq 45^{\circ}$ and $0^{\circ} \leq \phi \leq 45^{\circ}$, with special attentions to the $(\theta, \phi)$ regions where stable or unstable counter-rotating waves exist on $\mathrm{C}_{\mathrm{ub}}$ or $\mathrm{C}_{\mathrm{b}}$, and the $(\theta, \phi)$ region where stable co-rotating waves are observed on $\mathrm{C}_{\mathrm{ub}}$ for all $\delta$. We find that counter-rotating waves exist on $\mathrm{C}_{\mathrm{b}}$ only if $\phi \leq \phi_{\mathrm{b}}^{\text {all }}$ is satisfied, and that stable counter-rotating waves exist on $\mathrm{C}_{\mathrm{b}}$ for $\phi$ satisfying $\phi \leq \phi_{\mathrm{b}}^{\text {stable }}$. As expressed by $\bigcirc$ and $\square$ in figure 6 , boundary values $\phi_{\mathrm{b}}^{\text {all }}$ and $\phi_{\mathrm{b}}^{\text {stable }}$ decrease with increasing $\theta$. These values are the same for $0^{\circ} \leq \theta<15^{\circ}$ and $32^{\circ}<\theta \leq 45^{\circ}$, whereas the relation $\phi_{\mathrm{b}}^{\text {stable }}<\phi_{\mathrm{b}}^{\text {all }}$ holds for $15^{\circ}<\theta<32^{\circ}$. The maximum of $\phi_{\mathrm{b}}^{\text {all }}$ and $\phi_{\mathrm{b}}^{\text {stable }}$ is $43.96^{\circ}$ for $\theta=0^{\circ}$, and their minimum is $22.52^{\circ}$ for $\theta=45^{\circ}$. Moreover, if $\theta$ is larger than $28.9^{\circ}$, stable counter-rotating waves are observed on $\mathrm{C}_{\mathrm{ub}}$ for $\phi$ satisfying $\phi_{\mathrm{ub}}^{\min } \leq \phi \leq \phi_{\mathrm{ub}}^{\max }$. As shown in figure 6 by $\Delta$ and $\nabla, \phi_{\mathrm{ub}}^{\min }$ decreases to zero and $\phi_{\mathrm{ub}}^{\max }$ increases to $43.96^{\circ}$ as $\theta$ increases to $45^{\circ}$. Therefore, the region of $\phi$ where stable counter-rotating waves exist on $\mathrm{C}_{\mathrm{ub}}$ is the largest for $\theta=45^{\circ}$. It is also found that one or two stable co-rotating waves are observed on $\mathrm{C}_{\mathrm{ub}}$ for all $\delta$ if $\phi \geq \phi_{\mathrm{ub}}^{+}$ 

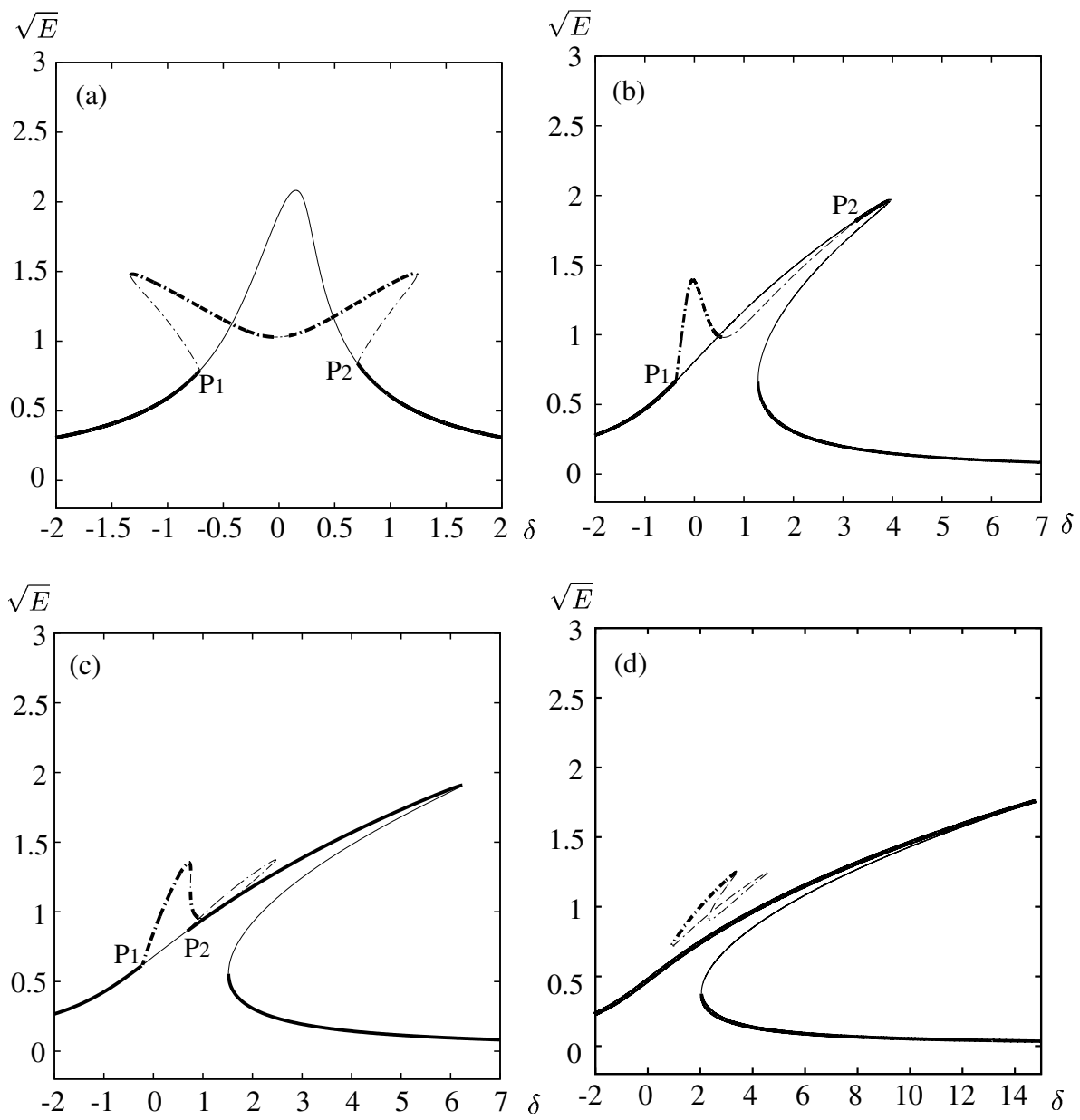

Figure 7. Response curves for $\alpha=0.3$ and $\theta=\phi=0^{\circ}$. (a) $h=\pi / 3$, (b) $h=0.857$, (c) $h=\pi / 4$ and (d) $h=\pi / 5$. Solid and dotted broken lines are stationary solutions with $M=0$ and $M \neq 0$, respectively. Thick and thin lines denote stable and unstable solutions, respectively. $\mathrm{P}_{1}$ and $\mathrm{P}_{2}$ denote pitchfork bifurcation points.

is satisfied. As shown in figure 6 , the value of $\phi_{\mathrm{ub}}^{+}$depends on $\theta$ only weakly, and takes the maximum of $37.40^{\circ}$ for $\theta=32.5^{\circ}$ and minimum of $33.78^{\circ}$ for $\theta=0^{\circ}$.

\subsubsection{Dependence on $h$}

The dependence of response curves of stationary solutions on depth $h$ is examined in this subsection only for a linear motion of the container with $\theta=\phi=0^{\circ}$ and $\alpha=0.3$. The coefficients of nonlinear terms in (15), $R_{1}, R_{2}$ and $R_{3}$, depend on $h$, as found from (16). It was shown by Nagata (1989) that $R_{1}$ is positive for sufficiently large $h$, decreases as $h$ decreases, and is negative for small $h$. Moreover, we find that $R_{3}$ is always positive and decreases as $h$ decreases, and that although $R_{2}$ can be positive or negative depending on $h,\left|R_{2}\right|$ is less than 0.1 for $h \geq \frac{\pi}{5}$. Response curves for $h=\frac{\pi}{3}, 0.857, \frac{\pi}{4}$ and $\frac{\pi}{5}$ are shown in figure 7 . The values of $R_{1}, R_{2}$ and $R_{3}$ for these $h$ 's and $h=\pi$ are shown in table 1 .

These response curves consist of a branch of non-rotating waves with $M=0$ and a branch of rotating waves resulting from a pair of solutions with $M>0$ and $M<0$ of 
Table 1. Values of $R_{1}, R_{2}$ and $R_{3}$ for a few $h$.

\begin{tabular}{cccc}
\hline$h$ & $R_{1}$ & $R_{2}$ & $R_{3}$ \\
\hline$\pi$ & 0.493 & $-4.52 \times 10^{-2}$ & 0.744 \\
$\pi / 3$ & $-1.37 \times 10^{-2}$ & $3.30 \times 10^{-2}$ & 0.450 \\
0.857 & -0.355 & $1.48 \times 10^{-2}$ & 0.359 \\
$\pi / 4$ & -0.561 & $-5.99 \times 10^{-3}$ & 0.323 \\
$\pi / 5$ & -1.33 & $-9.63 \times 10^{-2}$ & 0.240 \\
\hline
\end{tabular}

the same $\sqrt{E}$.

If $h$ is large enough to give positive $R_{1}$, the large $\sqrt{E}$ portion of the branch of nonrotating waves inclines toward the direction of decreasing $\delta$, as illustrated in figure 4(a) for $h=\pi$. However, if $h$ is $\frac{\pi}{3}$ for which $R_{1}$ is close to zero, this portion does not incline as found from figure 7(a). Moreover, if $h$ is small enough to give negative $R_{1}$, this portion inclines toward the direction of increasing $\delta$, as shown in figures $7(\mathrm{~b})-(\mathrm{d})$. This result can be explained as follows: If $B=0$ and $\frac{\partial A}{\partial \tau}=0$ are assumed in the first equation of (15) to consider the stationary solutions of non-rotating waves, we obtain

$$
\delta=-R_{1}|A|^{2} \pm \frac{\sqrt{\sin ^{2} \phi \sin ^{2} \theta+\cos ^{2} \phi \cos ^{2} \theta-\alpha^{2}|A|^{2}}}{|A|} .
$$

From the first term on the right-hand side of (18), we find that the sign of $R_{1}$ determines the direction of inclination of the large $\sqrt{E}$ portion of the branch of non-rotating waves. That is, because $\delta$ is close to $-R_{1}|A|^{2}$ at the peak of this portion where $|A|$ is close to $\frac{1}{\alpha}$ for $\theta=\phi=0^{\circ}$, this portion inclines toward the direction of decreasing (increasing) $\delta$ if $R_{1}>0\left(R_{1}<0\right)$. Moreover, it is expected that this portion inclines more strongly and extends to larger $|\delta|$ for larger $\left|R_{1}\right|$. This property is confirmed in figures $7(\mathrm{~b})-(\mathrm{d})$ if the dependence of $R_{1}$ on $h$ shown in table 1 is taken into account.

If $h$ is not small, the branch of rotating waves emerges from the branch of nonrotating waves through the pitchfork bifurcations at $\mathrm{P}_{1}$ and $\mathrm{P}_{2}$ shown in figure $4(\mathrm{a})$ and figures $7(\mathrm{a})-(\mathrm{c})$. For $h$ close to $\frac{\pi}{3}$, rotating waves are stable for $\delta$ in relatively large regions, as found from figure $7(\mathrm{a})$. If $h$ decreases from $\frac{\pi}{3}$, points $\mathrm{P}_{1}$ and $\mathrm{P}_{2}$ come closer to each other on the branch of non-rotating waves. Figure 7(b) for $h=0.857$ shows an example of the case in which point $\mathrm{P}_{2}$ is close to the peak of the large $\sqrt{E}$ portion of the branch of non-rotating waves as it moves along this branch toward $\mathrm{P}_{1}$. After the collision of these points, the branch of rotating waves separates from the branch of non-rotating waves, as shown in figure $7(\mathrm{~d})$. Although stable rotating waves may exist even after this separation as illustrated in this figure, it seems difficult to observe rotating waves in experiments for very small $h$ because they are isolated from the branch of non-rotating waves. Moreover, as found from figure $7(\mathrm{~d})$, we obtain one or two stable non-rotating waves for all $\delta$ for sufficiently small $h$ that gives negative $R_{1}$ of large modulus. 


\subsection{Non-stationary solutions}

For $\delta$ within the region of no stable stationary solutions, non-stationary solutions of (15) are computed numerically using the Adams method for fixed values of parameters, $\alpha=0.3, h=\pi$ and $\theta=0^{\circ}$.

\subsubsection{Dependence on $\phi$}

Figure 8 shows the dependence of Poincaré sections of non-stationary solutions on $\delta$ for $\phi=0^{\circ}, 10^{\circ}, 20^{\circ}$ and $30^{\circ}$. For these values of $\phi$, as found from figures $4(\mathrm{a})-(\mathrm{e})$, if $\delta$ is decreased from zero, stable stationary solutions on one or two branches of rotating waves are destabilized at the Hopf bifurcation points, some of which are denoted by $\mathrm{H}_{2}$ in these figures. In the computation of Poincaré sections, with the $\delta$ at these bifurcation points chosen as an initial value, $\delta$ is decreased slowly with decrement 0.001 . That is, for each $\delta$, only the solution during the period $2500 \leq \tau \leq 5000$ is used for Poincaré sections, with the solution for the last $\delta$ at $\tau=5000$ chosen as an initial value at $\tau=0$. Poincaré sections are produced by plotting the values of $M$ when non-stationary solutions cross the hyper surface $E=\langle E\rangle$ in the direction of decreasing $E$. Here $\langle E\rangle$ is the average of $E$ over the period $2500 \leq \tau \leq 5000$.

Figure 8(a) shows Poincaré sections for $\phi=0^{\circ}$ starting from a stationary solution with $M>0$. As $\delta$ decreases from -0.449 , which corresponds to $\mathrm{H}_{2}$ in figure 4(a), singly winding periodic orbits are first obtained as non-stationary solutions. With further decrease in $\delta$, these periodic orbits turn into doubly winding periodic orbits after the period-doubling bifurcation at $\mathrm{Pd}_{1}$ of figure 8(a), and then become chaotic through a series of period-doubling bifurcations. This transition process to chaos is similar to that obtained by Yoshimatsu and Funakoshi(2001) for infinite fluid depth. In the region of $\delta$ where chaotic solutions are mainly observed, many windows of periodic orbits are recognized. If $\delta$ is decreased from -0.8 , chaotic solutions return to simpler periodic orbits through a series of inverse period-doubling bifurcations, and singly winding periodic orbits appear again through the inverse period-doubling bifurcation at $\mathrm{Pd}_{2}$ of figure 8(a). For $-1.171 \leq \delta \leq-0.449$ (between $\mathrm{Sn}_{1}$ and $\mathrm{H}_{2}$ in figure 8(a)), the sign of $M$ of non-stationary solutions is positive in most of the time. In other words, surface waves almost always rotate in one direction, although their amplitudes and behavior of rotation of their pattern slowly vary in time. These waves are called uni-directionally rotating waves hereafter. If $\delta$ decreases from -1.171 , the periodic orbits of uni-directionally rotating waves disappear via the saddle-node bifurcation at $\mathrm{Sn}_{1}$ of figure 8(a), which causes a jump of solution to another periodic orbit. For $-1.478 \leq \delta \leq-1.172$, the sign of $M$ of non-stationary solutions varies in time, meaning that the direction of rotation of the wave pattern changes over time. These waves are called bi-directionally rotating waves hereafter. If $\delta$ is decreased from -1.172 , the periodic orbit of bi-directionally rotating waves that appears at $\mathrm{Sn}_{1}$ changes to chaotic solutions (with several small windows of periodic orbits) of bi-directionally rotating waves. As $\delta$ is decreased further, non-stationary solutions jump to a stable stationary solution on the branch of non- 

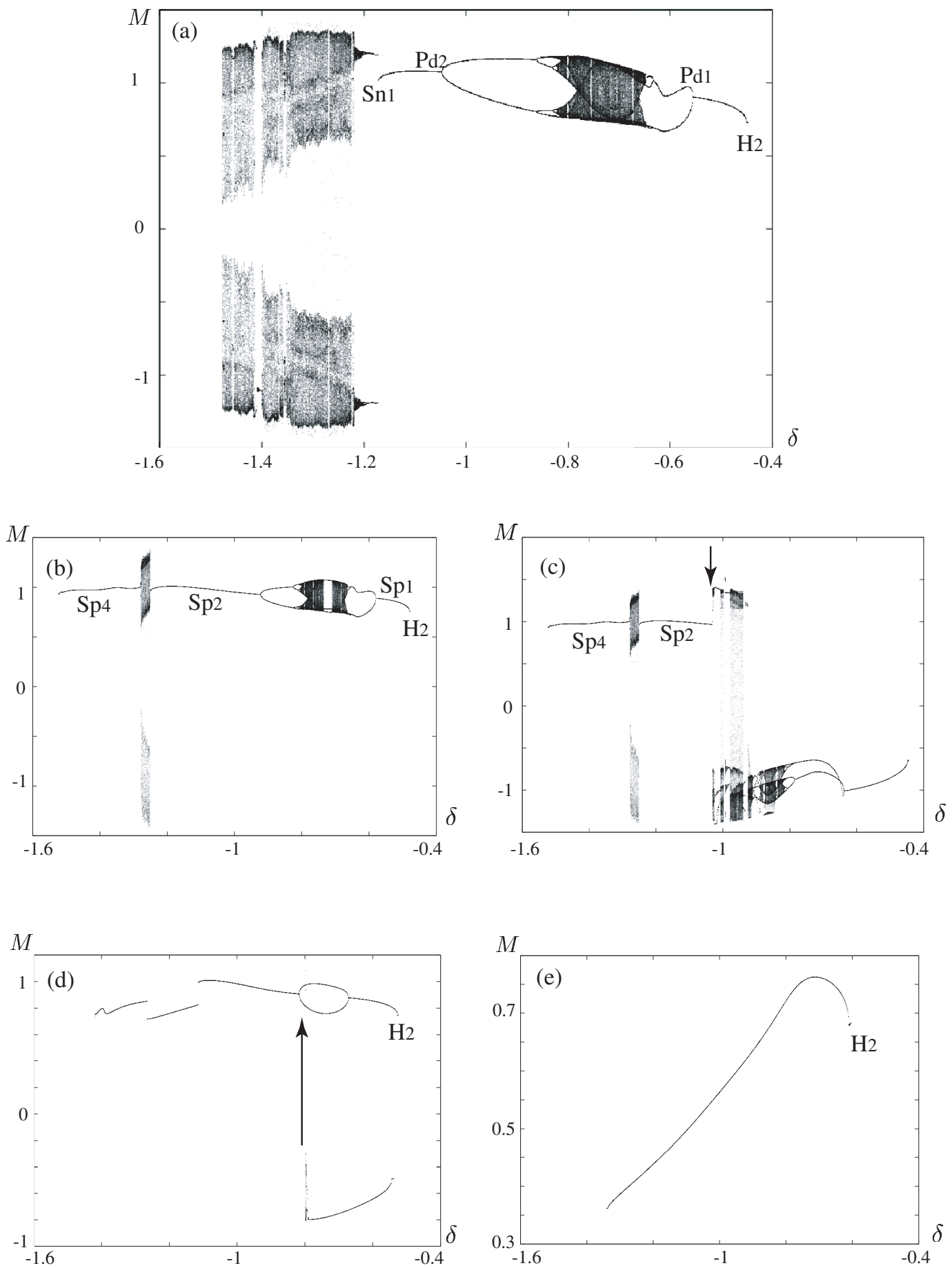

Figure 8. Poincaré sections for decreasing $\delta$ with decrement 0.001. $\alpha=0.3, h=\pi$ and $\theta=0^{\circ}$. (a) $\phi=0^{\circ}$, (b) and (c) $\phi=10^{\circ}$, (d) $\phi=20^{\circ}$ and (e) $\phi=30^{\circ}$. $\mathrm{H}_{2}, \operatorname{Pd}_{j}(j=1,2)$ and $\mathrm{Sn}_{1}$ denote the points of Hopf bifurcation, period-doubling bifurcation and saddle-node bifurcation, respectively. $\operatorname{Sp}_{j}(j=1,2$ and 4$)$ denote periodic orbits of uni-directionally rotating waves. 
rotating waves in figure $4(\mathrm{a})$ at $\delta=-1.479$, which is smaller than the $\delta$ at $\mathrm{S}$ of this figure. The transitions of non-stationary solutions with decreasing $\delta$ described above are again similar to those obtained by Yoshimatsu and Funakoshi (2001) for infinite fluid depth. It is noted that because of the symmetry of the system, the Poincaré section for $\phi=0^{\circ}$ starting from a stationary solution with $M<0$ is the same as figure 8 (a) under reflection with respect to the line $M=0$.

Figures $8(\mathrm{~b})$ shows Poincaré sections for $\phi=10^{\circ}$ starting from a stationary solution with $M>0$. If $\delta$ decreases from -0.480 that corresponds to the Hopf bifurcation point $\mathrm{H}_{2}$ on $\mathrm{C}_{\mathrm{ub}}$ of figure $4(\mathrm{c})$, periodic solutions of uni-directionally rotating waves denoted by $\mathrm{Sp}_{1}$ in figure 8 (b) are initially observed. Similarly to the case of $\phi=0^{\circ}$, as $\delta$ decreases further, we observe the appearance of chaotic solutions through a series of period-doubling bifurcations, and the subsequent return to singly winding periodic orbits denoted by $\mathrm{Sp}_{2}$ in figure 8(b) through a series of inverse period-doubling bifurcations. Although all of these solutions are uni-directionally rotating waves with $M>0$, the attractors that appear when $\delta$ decreases beyond -1.251 are chaotic solutions of bidirectionally rotating waves. However, the chaotic solutions are observed only for $\delta$ within the region $-1.278 \leq \delta \leq-1.251$, which is much smaller than the region of bidirectionally rotating waves for $\phi=0^{\circ}$. After the jump at $\delta=-1.279$ of the chaotic solutions to singly winding periodic orbits of uni-directionally rotating waves denoted by $\mathrm{Sp}_{4}$ in figure 8(b), stable stationary solutions with $M>0$ on $\mathrm{C}_{\mathrm{ub}}$ of figure $4(\mathrm{~b})$ are observed if $\delta$ decreases beyond -1.524, which is smaller than the $\delta$ at $\mathrm{S}$ of figure 4(b).

Figure $8(\mathrm{c})$ shows Poincaré sections for the same $\phi$ but starting from a stationary solution with $M<0$ at the Hopf bifurcation point on $\mathrm{C}_{\mathrm{b}}$ of figure 4(c), which gives slightly larger $\delta=-0.447$ than $\mathrm{H}_{2}$. As $\delta$ is decreased from this value, periodic orbits and then chaotic solutions of uni-directionally rotating waves with $M<0$ are obtained. Moreover, chaotic or periodic solutions of bi-directionally rotating waves are observed for $\delta$ around -1 , unlike the case of figure 8(b). However, at $\delta=-1.031$, these solutions jump to periodic orbits of uni-directionally rotating waves with $M>0$ that are the same as the periodic orbits denoted by $\mathrm{Sp}_{2}$ in figure $8(\mathrm{~b})$, as shown by an arrow in figure $8(\mathrm{c})$.

Figure $8(\mathrm{~d})$ shows a superposition of two Poincaré sections for $\phi=20^{\circ}$ starting from two stationary solutions with $M>0$ and $M<0$. Poincaré sections starting from a stationary solution with $M>0$ for $\delta=-0.526$ corresponding to $\mathrm{H}_{2}$ on $\mathrm{C}_{\mathrm{ub}}$ of figure 4(d) are composed of only periodic orbits of uni-directionally rotating waves with $M>0$, as shown in the part of positive $M$ in figure $8(\mathrm{~d})$. We also observe the jumps from a periodic orbit to another periodic orbit at $\delta=-1.115$ and -1.265 , which occur through saddle-node bifurcations. Moreover, as $\delta$ decreases beyond -1.420 , a jump from periodic orbits to stable stationary solutions with $M>0$ on $\mathrm{C}_{\mathrm{ub}}$ of figure $4(\mathrm{~d})$ is found. Poincaré sections starting from a stationary solution with $M<0$ for $\delta=-0.542$ corresponding to the Hopf bifurcation point on $\mathrm{C}_{\mathrm{b}}$ of figure $4(\mathrm{~d})$ are also composed of periodic orbits of uni-directionally rotating waves but with $M<0$ for $\delta$ larger than -0.798 , as shown in the part of negative $M$ in figure $8(\mathrm{~d})$. If $\delta$ is decreased beyond this 
value, these periodic orbits jump to a periodic orbit with $M>0$ that is found in the Poincaré sections starting from a stationary solution with $M>0$, as indicated by an arrow in figure $8(\mathrm{~d})$.

Figure $8(\mathrm{e})$ shows Poincaré sections for $\phi=30^{\circ}$ starting from a stationary solution with $M>0$ for $\delta=-0.609$ corresponding to $\mathrm{H}_{2}$ on $\mathrm{C}_{\mathrm{ub}}$ of figure $4(\mathrm{e})$. With the decrease in $\delta$, we observe only periodic orbits of uni-directionally rotating waves with $M>0$ until $\delta$ reaches -1.336 where a jump of periodic orbits to stable stationary solutions with $M>0$ on $\mathrm{C}_{\mathrm{ub}}$ of figure 4(e) is found.

From figure 8, we find the tendency that in a smaller region of $\delta$ non-stationary solutions of uni-directionally rotating waves with $M<0$ or bi-directionally rotating waves are observed for larger $\phi$. Moreover, the region of $\delta$ where chaotic solutions are observed becomes smaller as $\phi$ increases. In particular, for $\phi=20^{\circ}$ and $30^{\circ}$, neither chaotic solutions nor non-stationary solutions of bi-directionally rotating waves are obtained for most $\delta$.

\subsubsection{Poincaré sections starting from an almost quiescent state}

We also compute the Poincaré sections of non-stationary solutions starting from an almost quiescent state for each $\delta$. That is, initial values of variables in (15) for each $\delta$ are chosen as $A=B=(1+\mathrm{i}) \times 10^{-5}$. For $\phi=20^{\circ}$ and $30^{\circ}$, we find that the Poincaré sections for these initial values are almost the same as the part of positive $M$ in figure $8(\mathrm{~d})$ and figure $8(\mathrm{e})$, respectively. In contrast, for $\phi=10^{\circ}$, the Poincaré sections starting from an almost quiescent state shown in figure $9(\mathrm{a})$ are slightly different from figures $8(\mathrm{~b})$ and (c). That is, we observe periodic orbits denoted by $\mathrm{Sp}_{3}$ for $-0.785 \leq \delta \leq-0.576$ in figure 9(a), unlike the Poincaré sections of figures 8(b) and (c). Moreover, although a part of the transition from chaotic solutions to periodic orbits denoted by $\mathrm{Sp}_{2}$ in figure $8(\mathrm{~b})$ is reproduced in figure $9(\mathrm{a})$, chaotic solutions are often obtained for $\delta$ in the region $-1.119 \leq \delta \leq-0.982$ of figure $9(\mathrm{a})$, unlike the Poincaré sections of figure $8(\mathrm{~b})$. Some of these chaotic solutions may be the same as those shown in figure 8(c).

To understand some of the dependence of non-stationary solutions on $\delta$ for $\phi=10^{\circ}$ shown in the Poincaré sections of figures 8(b) and 9(a), the branch of singly winding periodic orbits with $M>0$ emerging through the Hopf bifurcation at $\mathrm{H}_{2}$ of figure $4(\mathrm{c})$ is computed numerically with the Newton's method. In figure 9(b), this branch is displayed in the same way as the Poincaré sections of non-stationary solutions. Periodic orbits denoted by $\operatorname{Sp}_{m}(m=1, \ldots, 4)$ in figures $8(\mathrm{~b})$ and $9(\mathrm{a})$ correspond to stable periodic orbits with the same names in figure $9(\mathrm{~b})$. Therefore, it is suggested that the domain of attraction of $\mathrm{Sp}_{3}$ is larger than that of stable periodic or chaotic solutions connecting $\mathrm{Sp}_{1}$ and $\mathrm{Sp}_{2}$ in figure 8(b), and that the domain of attraction of $\mathrm{Sp}_{2}$ is not large at least for $\delta$ around -1 . Moreover, it is found that the leftmost region of $\delta$ where chaotic solutions are obtained, $-1.279 \leq \delta \leq-1.251$, in figures 8 (b) and 9 (a) corresponds to a small gap in $\delta$ between $\mathrm{Sp}_{2}$ and $\mathrm{Sp}_{4}$ in figure $9(\mathrm{~b})$. Complicated dependence of periodic orbits and their stability on $\delta$ such as that shown in figure 9(b) may produce frequent transitions of observed non-stationary solutions with the variation in $\delta$ and complicated 

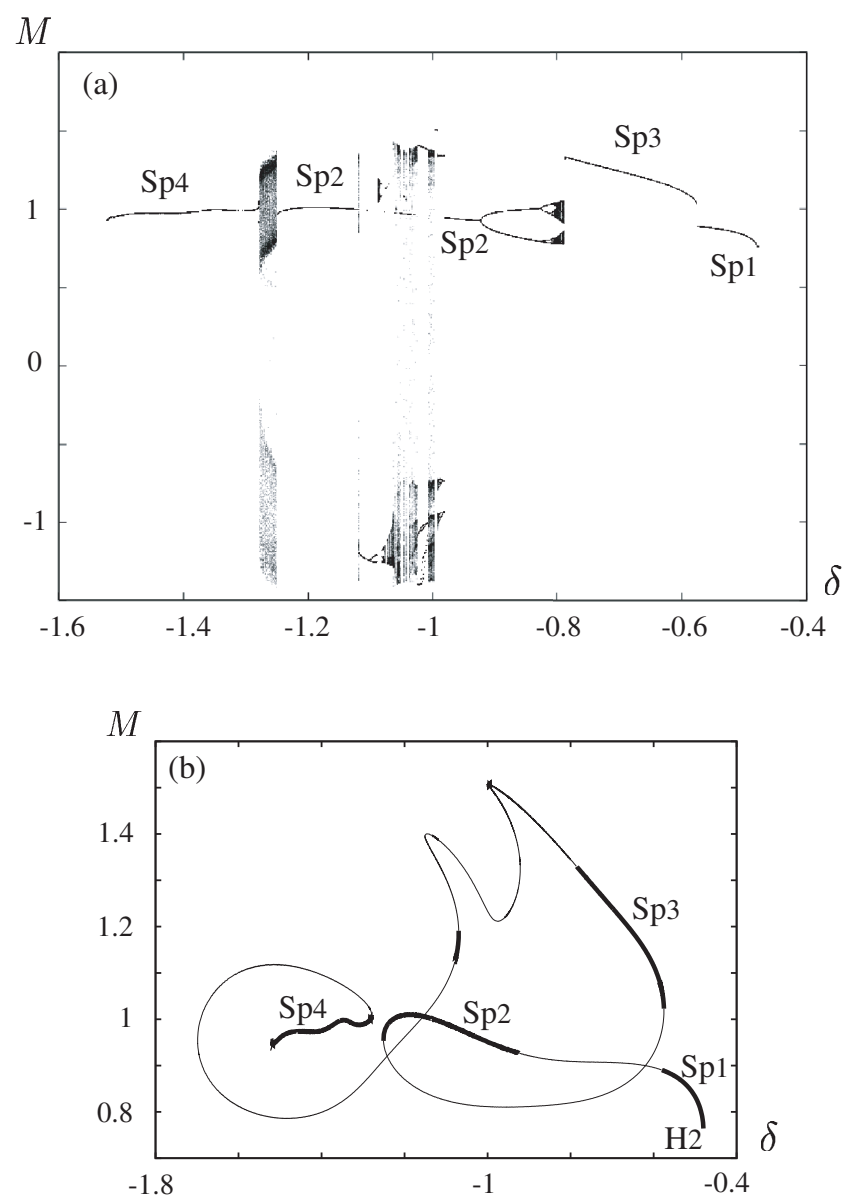

Figure 9. (a) Poincaré sections starting from an almost quiescent state for each $\delta$. $\alpha=0.3, h=\pi, \theta=0^{\circ}$ and $\phi=10^{\circ}$. (b) A part of the branch of periodic orbits starting from the Hopf bifurcation point $\mathrm{H}_{2}$. Thick and thin lines denote stable and unstable solutions, respectively. $\mathrm{Sp}_{j}(j=1, \ldots, 4)$ denote periodic orbits of uni-directionally rotating waves.

dependence of these solutions on initial conditions.

\section{Comparison with experiments}

Ikeda et al (2012) experimentally examined the excitation of surface waves in a rectangular container of square base with side length $100[\mathrm{~mm}]$ due to its resonant linear oscillation along three horizontal directions corresponding to $\theta=0^{\circ}, 30^{\circ}$ and $45^{\circ}$ of the present study. The fluid depth in their experiments is $60[\mathrm{~mm}]$, which gives the depth $h=1.885$. The natural frequency of two degenerate eigenmodes, $(1,0)$ and $(0,1)$ modes, of surface waves is $2.73[\mathrm{~Hz}]$. Ikeda et al $(2012)$ examined the excitation of surface waves with forcing frequency increased from $2.40[\mathrm{~Hz}]$, or decreased from $3.00[\mathrm{~Hz}]$ slowly. Moreover, they obtained the coefficient of linear damping by curve fitting of experimental data. Stationary and non-stationary solutions of (15) for $\phi=0^{\circ}$ and 
$h=1.885$ are compared with their experimental results.

In their experiment with $\theta=0^{\circ}$, the displacement amplitude of the container was $0.727[\mathrm{~mm}]$. The linear damping coefficient $\alpha$ in (15) corresponding to this experiment is determined as 0.403. Figure 10 shows the amplitudes of free-surface displacements $\eta_{x}$ and $\eta_{y}$ at points $\mathrm{M}_{1}$ and $\mathrm{M}_{2}$ that are located at a distance of $40[\mathrm{~mm}]$ from the center of the container in the $x$ and $y$ directions in our coordinate system, respectively, for several values of forcing frequency $\tilde{\Omega}[\mathrm{Hz}]$. The experimental data by Ikeda et al (2012) are shown as a few kinds of circles. Open circles denote non-rotating surface waves of time-independent amplitude, whereas half filled circles denote surface waves of time-independent amplitude that rotate anticlockwise. Moreover, solid circles denote the maxima of time-dependent amplitudes of surface waves. Amplitudes of $\eta_{x}$ and $\eta_{y}$ obtained from the stationary solutions of (15) for the values of parameters described above are shown as solid and dotted broken lines. Moreover, symbol + denotes the maxima of time-dependent amplitudes of $\eta_{x}$ and $\eta_{y}$ obtained from the non-stationary solutions of (15) for slowly decreasing forcing frequency. It is noted that almost the same maxima are obtained even when the forcing frequency is increased slowly.

We find that the agreement between experimental results and our theoretical results is fairly good. That is, the amplitudes of $\eta_{x}$ and $\eta_{y}$ obtained from stable stationary solutions of rotating or non-rotating waves agree well with the experimental data of timeindependent amplitudes of such waves for $\tilde{\Omega}$ between 2.69 and 3. For $\tilde{\Omega} \leq 2.62$, although there is some discrepancy between the experimental data on the wave amplitudes and the region of $\tilde{\Omega}$ where waves of time-independent amplitude are observed and our theoretical results obtained from stable stationary solutions of (15), we see qualitative agreement between them. The waves of larger amplitudes observed for $2.56<\tilde{\Omega}<2.63$ were described as non-rotating waves by Ikeda et al (2012), contrary to the prediction of rotating waves in our results. However, since theoretical values of $\left|\frac{M}{E}\right|$ of these rotating waves are smaller than 0.28 , these waves were thought to be non-rotating, as was explained in section 2.2. The above discrepancy between our theoretical results and experimental results may be partly caused by the use of weakly nonlinear theory. Another possible reason for this discrepancy is the difficulty in measuring the linear damping coefficient very precisely in the experiments, because the locations of saddlenode bifurcations $\mathrm{Sn}_{1}$ and $\mathrm{Sn}_{2}$ shown in figure 10 on the branches of non-rotating and rotating waves, respectively, depend fairly strongly on $\alpha$. In the experiments, with decreasing $\tilde{\Omega}$ from 2.69, rapid increase in the maxima of time-dependent amplitudes of $\eta_{x}$ and $\eta_{y}$ is observed until $\tilde{\Omega}$ reaches 2.63 , where a sudden change to the waves of time-independent amplitudes occurs. Our theoretical results obtained from nonstationary solutions of (15) similarly show a rapid increase in the maxima of timedependent amplitudes of $\eta_{x}$ and $\eta_{y}$ and a sudden change to time-independent amplitudes of stationary solutions with the decrease in $\tilde{\Omega}$ from 2.69 .

The experiment by Ikeda et al (2012) for $\theta=30^{\circ}$ was performed with an amplitude of $0.726[\mathrm{~mm}]$ in the container's displacement. Measured damping ratio corresponds to $\alpha=0.403$ in (15). In figure 11, measured amplitudes of $\eta_{x}$ and $\eta_{y}$ for several $\tilde{\Omega}$ 

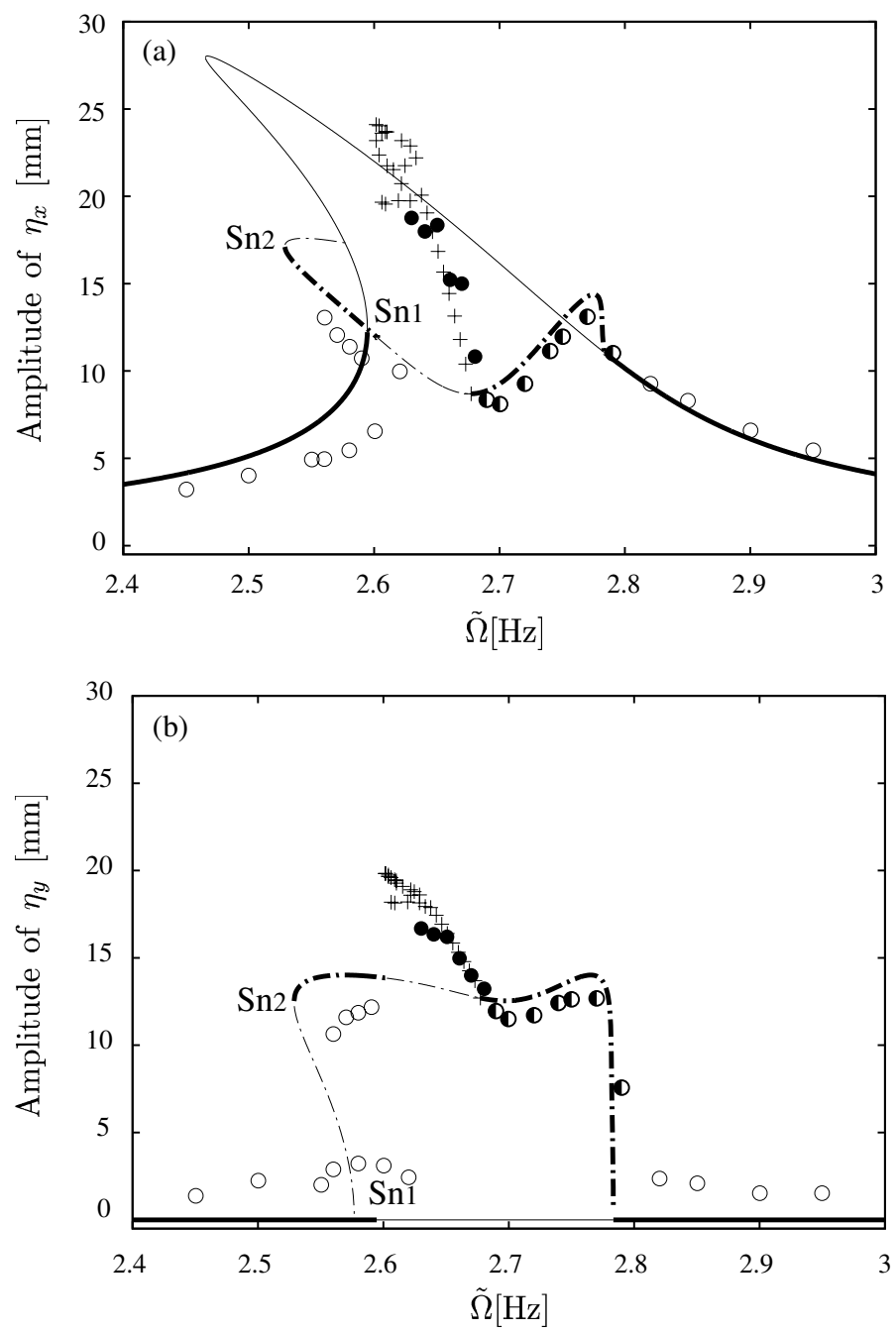

Figure 10. Comparison with the experimental data on amplitudes of free-surface displacements $\eta_{x}$ and $\eta_{y}$ at points $\mathrm{M}_{1}$ and $\mathrm{M}_{2}$, respectively. Circles are experimental data by Ikeda et al (2012). Open and half filled circles denote non-rotating and rotating surface waves of time-independent amplitude, respectively. Solid circles denote the maxima of time-dependent amplitudes of surface waves. Solid and dotted broken lines are amplitudes obtained from stationary solutions of (15) with $M=0$ and with $M \neq 0$, respectively, for $\theta=\phi=0^{\circ}, h=1.885$ and $\alpha=0.403$. Thick and thin lines denote stable and unstable solutions, respectively. + denotes the maxima of time-dependent amplitudes obtained from non-stationary solutions of (15).

are denoted by different kinds of circles. Here the definitions of solid and open circles are the same as those in figure 10, whereas left-filled (right-filled) circles denote surface waves of time-independent amplitude rotating counterclockwise (clockwise). Amplitudes obtained from stationary solutions of (15) with $M>0$ and $M<0$ are displayed as solid and broken lines, respectively. Although non-rotating waves of time-independent amplitude were experimentally observed for $\tilde{\Omega}<2.62$ and $\tilde{\Omega}>2.82$, rotating waves with $M>0$ are obtained for such $\tilde{\Omega}$ in our theoretical study. This discrepancy may again result from small theoretical values of $\left|\frac{M}{E}\right|$ less than 0.16 in these rotating waves. For 

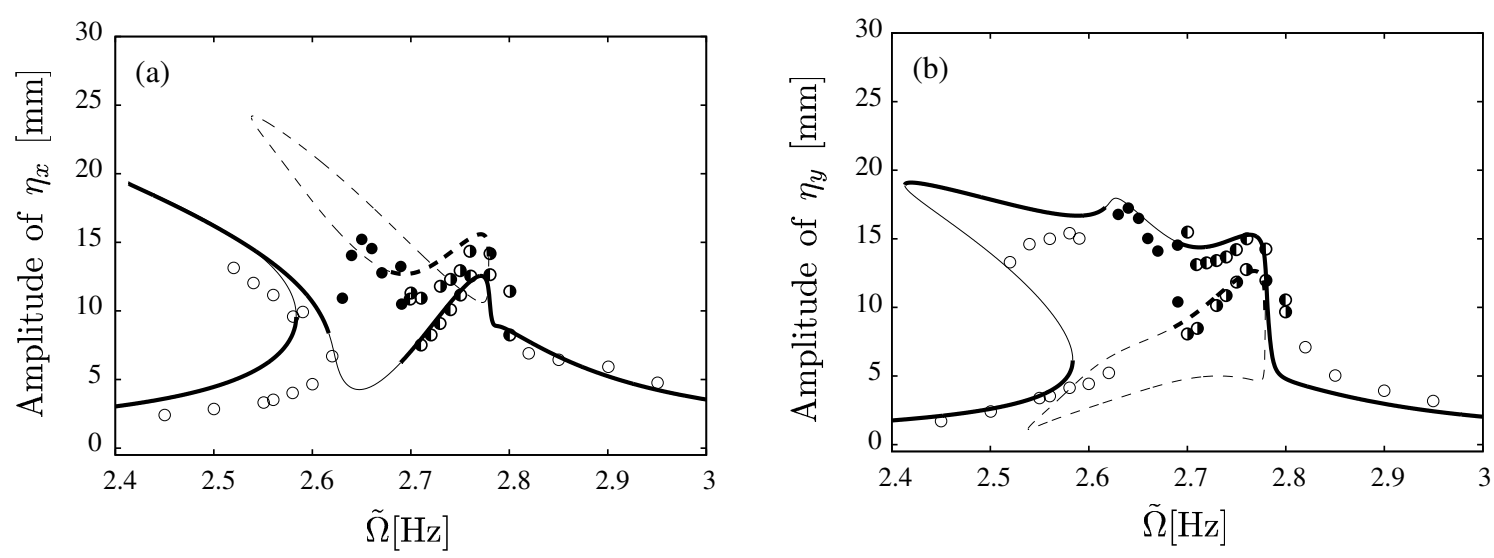

Figure 11. Comparison with the experimental data on amplitudes of free-surface displacements $\eta_{x}$ and $\eta_{y}$ at points $\mathrm{M}_{1}$ and $\mathrm{M}_{2}$, respectively. Circles are experimental data by Ikeda et al (2012). Open circles denote non-rotating surface waves of timeindependent amplitude, and left-filled (right-filled) circles denote surface waves of timeindependent amplitude rotating counterclockwise (clockwise). Solid circles denote the maxima of time-dependent amplitudes of surface waves. Solid and broken lines are amplitudes obtained from stationary solutions of (15) with $M>0$ and $M<0$, respectively, for $\theta=30^{\circ}, \phi=0^{\circ}, h=1.885$ and $\alpha=0.403$. Thick and thin lines denote stable and unstable solutions, respectively.

$\tilde{\Omega}$ within the region $2.70<\tilde{\Omega}<2.80$, both clockwise rotating waves of larger amplitude of $\eta_{x}$ and counterclockwise rotating waves of smaller amplitude of $\eta_{x}$ were observed in the experiments, depending on the direction of variation in $\tilde{\Omega}$. In our theoretical study, in almost the same region of $\tilde{\Omega}$, both stable stationary solutions with $M<0$ of larger amplitude of $\eta_{x}$ and those with $M>0$ of smaller amplitude of $\eta_{x}$ are obtained, similarly to the above experimental results. Moreover, the region of $\tilde{\Omega}$ within which timedependent amplitudes were observed in the experiments is close to the region where no stable stationary solution of (15) exists. However, the considerable extension of a part with large amplitude of $\eta_{x}$ toward $\tilde{\Omega}=2.41$ in stationary solutions with $M>0$ is not observed in the experiments.

Ikeda et al (2012) carried out experiments also for $\theta=45^{\circ}$, in which the displacement amplitude of the container was $0.717[\mathrm{~mm}]$. The linear damping coefficient $\alpha$ in (15) obtained from measured damping ratio is 0.406 . In figure 12 , amplitudes of $\eta_{x}$ and $\eta_{y}$ measured in the experiments are displayed as different kinds of circles whose definitions are the same as those in figure 11. Amplitudes obtained from stationary solutions of (15) with $M=0, M>0$ and $M<0$ are shown as solid, dotted broken and broken lines, respectively. From the symmetries of oscillated system and of two measurement points $\mathrm{M}_{1}$ and $\mathrm{M}_{2}$, the amplitude of $\eta_{x}\left(\eta_{y}\right)$ of solutions with $M>0$ is the same as that of $\eta_{y}\left(\eta_{x}\right)$ of solutions with $M<0$, and amplitudes of $\eta_{x}$ and $\eta_{y}$ are the same for solutions with $M=0$. The agreement between experimental and theoretical results on the time-independent amplitudes of $\eta_{x}$ and $\eta_{y}$ of waves rotating counterclockwise or clockwise for $\tilde{\Omega}$ between 2.69 and 2.78 is fairly good. The region of $\tilde{\Omega}$ just below 2.69 

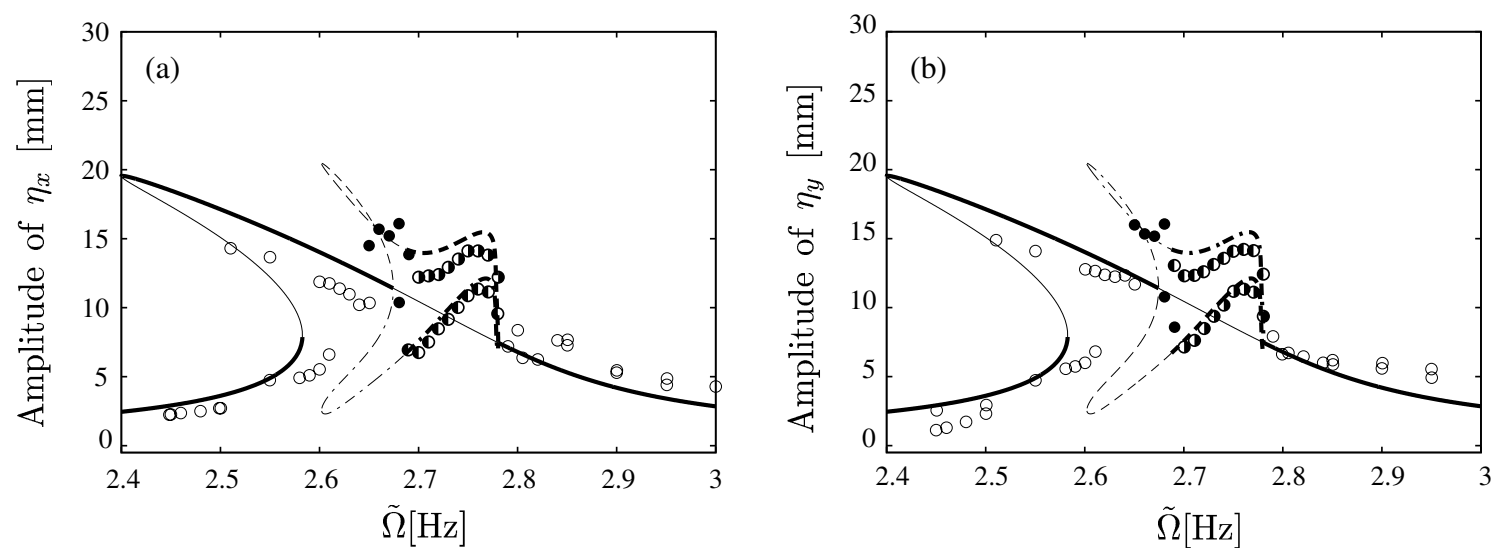

Figure 12. Comparison with the experimental data on amplitudes of free-surface displacements $\eta_{x}$ and $\eta_{y}$ at points $\mathrm{M}_{1}$ and $\mathrm{M}_{2}$, respectively. Circles are experimental data by Ikeda et al (2012). Open circles denote non-rotating surface waves of timeindependent amplitude, and left-filled (right-filled) circles denote surface waves of timeindependent amplitude rotating counterclockwise (clockwise). Solid circles denote the maxima of time-dependent amplitudes of surface waves. Solid lines are amplitudes obtained from stationary solutions of (15) with $M=0$, dotted broken lines are those with $M>0$ and broken lines are those with $M<0$ for $\theta=45^{\circ}, \phi=0^{\circ}, h=1.885$ and $\alpha=0.406$. Thick and thin lines denote stable and unstable solutions, respectively.

where rotating waves of time-dependent amplitude are observed in the experiments is close to the small region of no stable stationary solutions of (15). Moreover, amplitudes of $\eta_{x}$ and $\eta_{y}$ obtained from the stationary solutions with $M=0$ agree fairly well with amplitudes of non-rotating waves observed in the experiments, except for the extension of a part with large amplitudes of $\eta_{x}$ and $\eta_{y}$ toward $\tilde{\Omega}=2.40$ in these solutions.

From the above comparisons between our theoretical results and experimental results by Ikeda et al (2012), we can say that the wave amplitudes at two measurement points and the rotation of the wave pattern for several forcing frequencies observed in this experiment agree fairly well with our results. Moreover, the agreement is noticeably better for positive detuning than for negative detuning. Although this is an interesting tendency, the reason for it is not clear.

\section{Conclusions}

Three-dimensional surface waves in a square container due to its resonant horizontal elliptic or linear motion are investigated theoretically. The motion of the container is characterized by the ratio, expressed as $\tan \phi$, of the length of the minor axis to the length of the major axis of its elliptic orbit, and by the angle $\theta$ between the directions of the major axis and one of its sidewalls. We derive nonlinear evolution equations (15) for complex amplitudes of two degenerate modes excited by this motion, called $(1,0)$ and $(0,1)$ modes, using the reductive perturbation method and including the effect of linear damping. The parameters in these equations are the linear damping coefficient $\alpha$, the detuning $\delta$ between forcing frequency and eigenfrequency of these modes, and the 
fluid depth $h$, in addition to $\phi$ and $\theta$.

For fixed values of $\alpha$ and $h$, response curves, expressing the dependence on $\delta$ of a measure of the amplitude of the waves, $\sqrt{E}$, defined in section 2.2 , for stationary solutions to these equations, are computed numerically for several $\theta$ and $\phi$. Stationary solutions are characterized by the value of $M$ introduced in section 2.2, whose sign designates the direction of rotation of the wave pattern, as well as the value of $\sqrt{E}$. Non-rotating waves with $M=0$ are possible only for linear motions of the container with $\phi=0^{\circ}$ along the direction of $\theta=0^{\circ}$ or $45^{\circ}$. For other values of $\phi$ and $\theta$ satisfying $0^{\circ}<\phi \leq 45^{\circ}$ and $0^{\circ}<\theta<45^{\circ}$, only rotating waves with $M \neq 0$ can be observed. Moreover, for the elliptic motion of the container with $\phi \neq 0^{\circ}$, these waves are classified into co-rotating waves of $M>0$, whose direction of rotation is the same as that of the container, and counter-rotating waves of $M<0$, whose direction of rotation is opposite to that of the container's motion. Response curves for such $\phi$ are composed of an unbounded branch extending to $\delta \rightarrow \pm \infty$, and a bounded branch of a closed curve observed within a finite range of $\delta$ not far from zero, if $\phi$ is sufficiently small. With increasing $\phi$, this bounded branch shrinks, and the region of $\delta$ where stable counterrotating waves are observed on an unbounded or bounded branch becomes smaller for any $\theta$. If $\phi$ is sufficiently close to $45^{\circ}$ corresponding to a nearly circular motion of the container, stable co-rotating waves are observed for all $\delta$ on an unbounded branch for any $\theta$.

The threshold value of $\phi, \phi_{\mathrm{ub}}^{+}$, above which stable co-rotating waves exist for all $\delta$ depends on $\theta$ only weakly and is about $35^{\circ}$. This value of $\phi$ implies the ratio 0.7 of the lengths of two axes of elliptic motion. Stable counter-rotating waves are observed on a bounded branch only if $\phi$ is smaller than a threshold value $\phi_{\mathrm{b}}^{\text {stable }}$, which decreases from $43.96^{\circ}$ to $22.52^{\circ}$ as $\theta$ increases from $0^{\circ}$ to $45^{\circ}$. Moreover, if $\theta$ is larger than $28.9^{\circ}$, stable counter-rotating waves exist also on an unbounded branch for $\phi$ within the range of $\phi_{\mathrm{ub}}^{\min } \leq \phi \leq \phi_{\mathrm{ub}}^{\max }$. This range expands as $\theta$ increases from $28.9^{\circ}$ to $45^{\circ}$, and $\phi_{\mathrm{ub}}^{\max }$ is $43.96^{\circ}$ and $\phi_{\mathrm{ub}}^{\min }$ is $0^{\circ}$ for $\theta=45^{\circ}$.

Response curves are also computed for a few $h$ for a linear motion of the container with $\theta=\phi=0^{\circ}$. The large $\sqrt{E}$ portion of the response curve of non-rotating waves inclines toward the direction of decreasing $\delta$ if $h$ is sufficiently large. However, this portion inclines toward the opposite direction for small $h$, and inclines more strongly and extends to larger $\delta$ as $h$ decreases. Moreover, with decreasing $h$, the distance on the response curve of non-rotating waves between two pitchfork bifurcation points at which the response curve of rotating waves emerges decreases to zero, which causes the separation of response curves of rotating and non-rotating waves. Furthermore, the observation of regular non-rotating waves is expected for all $\delta$ if $h$ is sufficiently small.

For $\theta=0^{\circ}$, solutions with chaotic or periodic slow variations in the amplitude and phase of excited surface waves are observed for $\delta$ within the region of no stable stationary solutions. Non-stationary solutions are either uni-directionally or bi-directionally rotating waves. Chaotic solutions are observed more frequently for smaller $\phi$. Moreover, with increasing $\phi$, the region of $\delta$ where chaotic or periodic solutions of bi-directionally 
rotating waves are observed tends to shrink and then disappears. Only periodic solutions of uni-directionally rotating waves are observed for all the $\delta$ within the region of no stable stationary solutions if $\phi$ is sufficiently large. Some of the transitions of non-stationary solutions associated with the variation in $\delta$ and the multiplicity of these solutions can be explained from a complicated branching of periodic solutions.

Finally, stable stationary and non-stationary solutions for the linear motion of the container with $\theta=0^{\circ}, 30^{\circ}$ and $45^{\circ}$ are compared with the experimental results by Ikeda et al (2012). The wave amplitudes at two measurement points and the rotation of the wave pattern for several forcing frequencies observed in this experiment agree fairly well with our results.

\section{Acknowledgments}

This work was supported by JSPS KAKENHI Grant Number 25390154.

\section{Appendix A. Equations at $O\left(\epsilon^{2}\right)$ and $O\left(\epsilon^{3}\right)$}

Equations obtained at $O\left(\epsilon^{2}\right)$ of (7) and (8) are

$$
\left\{\begin{array}{l}
\partial_{t} \Phi_{2}+\eta_{2}+\eta_{1} \partial_{z} \partial_{t} \Phi_{1}+\frac{1}{2}\left(\nabla \Phi_{1}\right)^{2}=0, \quad \text { at } z=0, \\
\partial_{t} \eta_{2}-\partial_{z} \Phi_{2}-\eta_{1} \partial_{z}^{2} \Phi_{1}+\left(\partial_{x} \eta_{1}\right)\left(\partial_{x} \Phi_{1}\right)+\left(\partial_{y} \eta_{1}\right)\left(\partial_{y} \Phi_{1}\right)=0, \quad \text { at } z=0,
\end{array}\right.
$$

whereas equations obtained at $O\left(\epsilon^{3}\right)$ of the same equations are written as

$$
\left\{\begin{aligned}
\partial_{t} \Phi_{3} & +\eta_{3}+\partial_{\tau} \Phi_{1}+\pi\{\cos \phi \cos \theta \cos (H t+\delta \tau)-\sin \phi \sin \theta \sin (H t+\delta \tau)\} x \\
& +\pi\{\cos \phi \sin \theta \cos (H t+\delta \tau)+\sin \phi \cos \theta \sin (H t+\delta \tau)\} y+\eta_{1} \partial_{z} \partial_{t} \Phi_{2}+\eta_{2} \partial_{z} \partial_{t} \Phi_{1} \\
& +\frac{1}{2} \eta_{1}^{2} \partial_{z}^{2} \partial_{t} \Phi_{1}+\left(\nabla \Phi_{1}\right) \cdot\left(\nabla \Phi_{2}\right)+\frac{1}{2} \eta_{1} \partial_{z}\left[\left(\nabla \Phi_{1}\right)^{2}\right]=0, \quad \text { at } z=0, \\
\partial_{t} \eta_{3} & -\partial_{z} \Phi_{3}+\partial_{\tau} \eta_{1}-\eta_{1} \partial_{z}^{2} \Phi_{2}-\eta_{2} \partial_{z}^{2} \Phi_{1}-\frac{1}{2} \eta_{1}^{2} \partial_{z}^{3} \Phi_{1} \\
& +\left(\partial_{x} \eta_{1}\right)\left(\partial_{x} \Phi_{2}\right)+\left(\partial_{x} \eta_{2}\right)\left(\partial_{x} \Phi_{1}\right)+\eta_{1}\left(\partial_{x} \eta_{1}\right)\left(\partial_{z} \partial_{x} \Phi_{1}\right) \\
& +\left(\partial_{y} \eta_{1}\right)\left(\partial_{y} \Phi_{2}\right)+\left(\partial_{y} \eta_{2}\right)\left(\partial_{y} \Phi_{1}\right)+\eta_{1}\left(\partial_{y} \eta_{1}\right)\left(\partial_{z} \partial_{y} \Phi_{1}\right)=0, \quad \text { at } z=0 .
\end{aligned}\right.
$$

Substituting (14) into (A.1) and using (13) with $j=2$, we obtain

$$
\left\{\begin{aligned}
\Phi_{2}= & \mathrm{i} S_{1}\left(a^{2}+b^{2}\right) \mathrm{e}^{2 \mathrm{i} H t}+\mathrm{i} S_{2} a b \mathrm{e}^{2 \mathrm{i} H t} \cos x \cos y \frac{\cosh [\sqrt{2}(z+h)]}{\cosh (\sqrt{2} h)} \\
& +\mathrm{i} S_{3}\left(a^{2} \cos 2 x+b^{2} \cos 2 y\right) \mathrm{e}^{2 \mathrm{i} H t} \frac{\cosh [2(z+h)]}{\cosh (2 h)}+\text { c.c. } \\
\eta_{2}= & \left(S_{4} a b \mathrm{e}^{2 \mathrm{i} H t}+S_{5} a b^{*}\right) \cos x \cos y+\left(S_{6} a^{2} \mathrm{e}^{2 \mathrm{i} H t}+S_{7}|a|^{2}\right) \cos 2 x \\
& +\left(S_{6} b^{2} \mathrm{e}^{2 \mathrm{i} H t}+S_{7}|b|^{2}\right) \cos 2 y+\text { c.c. }
\end{aligned}\right.
$$


where $*$ denotes complex conjugate, and $S_{1}, S_{2}, \ldots, S_{7}$ are given by

$$
\left\{\begin{array}{l}
S_{1}=\frac{1}{8 H}\left(3 H^{4}+1\right), \quad S_{2}=\frac{2 H}{4 H^{2}-\sqrt{2} \tanh (\sqrt{2} h)}\left(3 H^{4}-1\right), \\
S_{3}=\frac{3 H}{4\left(2 H^{2}-\tanh (2 h)\right)}\left(H^{4}-1\right), \\
S_{4}=\frac{H^{2}}{4 H^{2}-\sqrt{2} \tanh (\sqrt{2} h)}\left(3 \sqrt{2} \tanh (\sqrt{2} h) H^{2}-4\right), \quad S_{5}=H^{4}, \\
S_{6}=\frac{\left(3 H^{4}-1\right) \tanh (2 h)-4 H^{2}}{4\left(2 H^{2}-\tanh (2 h)\right)}, \quad S_{7}=\frac{1}{4}\left(H^{4}+1\right) .
\end{array}\right.
$$

\section{References}

Faltinsen O M and Timokha A N 2009 Sloshing (Cambridge: Cambridge University Press).

Funakoshi M and Inoue S 1988 Surface waves due to resonant horizontal oscillation. J. Fluid Mech. 192, 219-247.

Funakoshi M and Inoue S 1990 Bifurcations of limit cycles in surface waves due to resonant forcing. Fluid Dyn. Res. 5, 255-271.

Hutton R E 1963 An investigation of resonant, nonlinear, nonplaner free surface oscillations of a fluid. NASA Tech. Note D-1870, 1-64.

Ibrahim R A 2005 Liquid Sloshing Dynamics (Cambridge: Cambridge University Press).

Ikeda T, Ibrahim R A, Harata Y and Kuriyama T 2012 Nonlinear liquid sloshing in a square tank subjected to obliquely horizontal excitation. J. Fluid Mech. 700, 304-328.

Miles J W 1984 Resonantly forced surface waves in a circular cylinder. J. Fluid Mech. 149, 15-31.

Miles J and Henderson D 1990 Parametrically forced surface waves. Annu. Rev. Fluid Mech. 22, 143165.

Nagata M 1989 Nonlinear Faraday resonance in a box with a square base. J. Fluid Mech. 209, 265-284.

Nobili M, Ciliberto S, Cocciaro B, Faetti S and Fronzoni L 1988 Time-dependent surface waves in a horizontally oscillating container. Europhys. Lett 7, 587-592.

Royon-Lebeaud A, Hopfinger E J and Cartellier A 2007 Liquid sloshing and wave breaking in circular and square-base cylindrical containers. J. Fluid Mech. 577, 467-494.

Yoshimatsu K and Funakoshi M 2001 Surface waves in a square container due to resonant horizontal oscillations. J. Phys. Soc. Jpn. 70, 394-406. 